\title{
Implementing The Simplex Method: The Initial Basis
}

\author{
Robert E. Bixby ${ }^{1}$ \\ Department of Mathematical Sciences \\ Rice University \\ September 1991 \\ (revised) \\ TR90-32
}

\footnotetext{
${ }^{1}$ The preparation of this manuscript was supported in part by NSF grant CCR-8815914 and AFOSR grant AFOSR-90-0273, both to Rice University.
} 



\begin{abstract}
This paper contains the first two parts of a planned series of papers on the CPLEX ${ }^{2}$ implementation of the simplex method. Part I is introductory. It gives an elementary description of the bounded-variable simplex method as well as a rather detailed discussion of some of the numerical characteristics of the netlib test problems. These problems form the basis for the computational tests in the subsequent parts. Part II contains the main results of this paper, a description of the method used by CPLEX for constructing an initial basis.
\end{abstract}

\footnotetext{
${ }^{2}$ CPLEX is a trademark of CPLEX Optimization, Inc.
} 



\section{Part I: Introduction}

\section{Introduction}

Ten years ago, linear programming was considered a computationally mature subject. Dantzig's simplex method [3] was thought to be well understood, and there were several state-of-the-art implementations of that method and its extensions; moreover, it is probable that the majority of the mathematical-programming community did not expect substantial improvements. That expectation was incorrect. The last ten years have seen dramatic changes in computing machinery. These changes have allowed a wider variety of strategies to be implemented and much larger problems to be studied in detail. Developments have also been driven by demands from other areas of optimization, particularly integer programming, where the availability of a good linear optimizer is essential. In addition, motivated by work of Karmarkar [6], a new class of methods, interior-point methods, has emerged as an alternative to the simplex method. Although interior-point methods are not treated in this paper, the recent computational results have been every bit as impressive as those for the simplex method ([1], [9]).

This paper is the first in a series of papers describing different aspects of the CPLEX implementation of the simplex method, the optimization routines for which were written by this author. Two other excellent, recent implementations of the simplex method are the OSL (Optimization System Library) implementation [5], written by John Forrest, and MINOS 5.3, written by Michael Saunders [11], the latter being a significant improvement over earlier versions of MINOS.

The main purpose of the present paper, and the subject of Part II, is to describe the procedure used by CPLEX for constructing a good initial basis. Part I provides a foundation for that discussion. It includes the definition of a basis and a statement of the bounded-variable simplex method, followed by a discussion of the numerical properties of the netlib test problems. Some readers more familiar with linear programming computation may wish to skip directly to Part II.

In subsequent papers in this series, other CPLEX features will be discussed, including the use of Philip Wolfe's composite simplex method in phase I, an inexpensive partial-pricing procedure, a simple bound-perturbation for dealing with stalling, vectorization and other topics. 


\section{The Definition of a Basis}

A linear programming problem (LP) is an optimization problem of the form

$$
\begin{array}{ll}
\operatorname{minimize} & c^{T} x \\
\text { s.t. } & A x=b \\
& l \leq x \leq u
\end{array}
$$

where $c, A, b, l$ and $u$ are given matrices with dimensions $n \times 1, m \times n, m \times 1, n \times 1$ and $n \times 1$, respectively, and $x$ is an $n \times 1$ vector of variables. Assume that $A$ has full row rank. When this condition is not met, appropriate unit columns can be added.

The linear functional $c^{T} x$ is called the objective function, $A$ the constraint matrix, $b$ the right-hand side, $l$ the vector of lower bounds and $u$ the vector of upper bounds. The "values" $-\infty$ for lower bounds and $+\infty$ for upper bounds are allowed. Most $x_{j}$ have $l_{j}=0$ and $u_{j}=+\infty$, and are called nonnegative. If $l_{j}=-\infty$ and $u_{j}=+\infty, x_{j}$ is free, and if $l_{j}=u_{j}, x_{j}$ is fixed.

If $S$ a subset of columns of $A, A_{S}$ denotes "the" $m \times|S|$ submatrix containing the columns in $S$. If $S$ is an ordered set, the columns of $A_{S}$ are taken to appear in this order. If $d$ is a vector and $S$ is a subset of row indices, then $d_{S}$ is the corresponding subvector. Again, if $S$ is ordered, the rows of $d_{S}$ are taken ordered.

Definition. A basis is a triple $\left(B, N_{l}, N_{u}\right)$ with the following two properties:

(B1) $B=\left(B_{1}, \ldots, B_{m}\right) \subseteq\{1, \ldots, n\}$ is an ordered subset of column indices such that $\boldsymbol{B}=A_{B}$ is nonsingular. $B$ is called the basis header and $\boldsymbol{B}$ the basis matrix. The variables $x_{j}(j \in B)$ are called basic variables. The remaining variables are nonbasic. $N=\{j: j \notin B\}$ denotes the set of indices of nonbasic variables.

(B2) $N_{l} \cap N_{u}=\emptyset, N_{l} \cup N_{u}=\left\{j \notin B: x_{j}\right.$ neither fixed nor free $\}, l_{j}>-\infty$ for $j \in N_{l}$ and $u_{j}<+\infty$ for $j \in N_{u}$.

Denote $N_{f r}=\left\{j \in N: x_{j}\right.$ free $\}$ and $N_{f x}=\left\{j \in N: x_{j}\right.$ fixed $\}$.

The triple $\left(B, N_{l}, N_{u}\right)$ is sometimes referred to as "the basis B." The set $B$ is assumed ordered to simplify the statement of the algorithm. There is no corresponding need to order $N_{l}$ or $N_{u}$.

Corresponding to each basis there is a basic solution $X$ given by

$$
\begin{aligned}
& X_{N_{l}}=l_{N_{l}}, \\
& X_{N_{u}}=u_{N_{u}}, \\
& X_{N_{f x}}=l_{N_{f x}}=u_{N_{f x}}, \\
& X_{N_{f r}}=0, \text { and } \\
& X_{B}=B^{-1}\left(b-A_{N} X_{N}\right) .
\end{aligned}
$$

The basis $B$ is called feasible if $l_{B} \leq X_{B} \leq u_{B}$. 


\section{The Bounded-Variable Simplex Method}

Algorithm 3.1. A Single Iteration of a Simplex Algorithm for (1)

Input: A feasible basis $B$ and the corresponding values $X_{B}$ of the basic variables.

Step S1: Solve $\pi^{T} \boldsymbol{B}=c_{B}^{T}$ for $\pi$.

Step S2: (Pricing) Compute all or part of $d_{N}=c_{N}-A_{N}^{T} \pi$. If $d_{j} \geq 0$ for all $j \in N_{l}, d_{j} \leq 0$ for all $j \in N_{u}$ and $d_{j}=0$ for all $j \in N_{f r}$, stop- $B$ is optimal; otherwise, select an entering variable $x_{j_{e}}, j_{e} \in N$, such that $d_{j_{e}}$ violates these conditions.

Step S3: Solve $B y=A_{j_{e}}$.

Step $S_{4}$ : (Ratio Test) If $d_{j_{e}}<0$, let

$$
\Theta_{i}=\left\{\begin{array}{cl}
+\infty & \text { if } y_{i}=0, \\
\left(X_{B_{i}}-l_{B_{i}}\right) / y_{i} & \text { if } \quad y_{i}>0, \text { and } \\
\left(X_{B_{i}}-u_{B_{i}}\right) / y_{i} & \text { if } \quad y_{i}<0,
\end{array}\right.
$$

and if $d_{j_{e}}>0$, let

$$
\Theta_{i}=\left\{\begin{array}{cl}
+\infty & \text { if } y_{i}=0, \\
\left(u_{B_{i}}-X_{B_{i}}\right) / y_{i} & \text { if } y_{i}>0, \text { and } \\
\left(l_{B_{i}}-X_{B_{i}}\right) / y_{i} & \text { if } y_{i}<0
\end{array}\right.
$$

for $i=1, \ldots, m$. Let

$$
\Theta=\min \left\{\min _{i} \Theta_{i}, u_{j_{e}}-l_{j_{e}}\right\}
$$

If $\Theta=+\infty$, stop-(1) is unbounded.

Step S5: (Update) If $d_{j_{e}}<0$, set $X_{B} \leftarrow X_{B}-\Theta y$; otherwise, set $X_{B} \leftarrow$ $X_{B}+\Theta y$.

(S5.1) For $\Theta=u_{j_{e}}-l_{j_{e}}$ : If $j_{e} \in N_{u}$, set $N_{l} \leftarrow N_{l} \cup\left\{j_{e}\right\}$ and $N_{u}^{\prime} \leftarrow$ $N_{u} \backslash\left\{j_{e}\right\}$; otherwise, set $N_{u} \leftarrow N_{u} \cup\left\{j_{e}\right\}$ and $N_{l} \leftarrow N_{l} \backslash\left\{j_{e}\right\}$.

(S5.2) For $\Theta<u_{j_{e}}-l_{j_{e}}$ : Let $i_{l} \in B$ be such that $\Theta_{i_{l}}=\Theta$, and let $j_{l}=B_{i_{l}}$ $\left(x_{j_{l}}\right.$ is the leaving variable). Set $B_{i_{l}} \leftarrow j_{e}$, and set

$$
X_{B_{i_{l}}} \leftarrow\left\{\begin{array}{cl}
l_{j_{e}}+\Theta & \text { if } j_{e} \in N_{l}, \\
u_{j_{e}}-\Theta & \text { if } j_{e} \in N_{u}, \\
\Theta & \text { if } j_{e} \in N_{f r} \text { and } d_{j_{e}}<0, \text { and } \\
-\Theta & \text { if } j_{e} \in N_{f r} \text { and } d_{j_{e}}>0 .
\end{array}\right.
$$

If $x_{j_{l}}$ is fixed, set $N_{f x} \leftarrow N_{f x} \cup\left\{j_{l}\right\}$; otherwise, if $d_{j_{e}} y_{i_{l}}<0$, set $N_{l} \leftarrow N_{l} \cup\left\{j_{l}\right\}$, and if $d_{j_{e}} y_{i_{l}}>0$, set $N_{u} \leftarrow N_{u} \cup\left\{j_{l}\right\}$. Remove $j_{e}$ from $N_{f r} \cup N_{l} \cup N_{u}$. 
Problem (1) is unbounded if for an arbitrary integer $M$, there is an $x$ feasible for (1) such that $c^{T} x<M$. A basis $B$ is optimal for (1) if it is feasible and $c^{T} X \leq c^{T} x$ for all feasible $x$, where $X$ is the basic solution corresponding to $B$.

The following theorem gives all the properties of Algorithm 3.1 that are necessary to deduce it's validity, namely: that it can be applied iteratively and that its termination criteria are correct. In addition, it is noted that the sequence of objectivefunction values produced is monotone. On the other hand, finite termination is not proved, nor is that conclusion valid for the given statement of the algorithm [2].

Theorem. If Algorithm 3.1 terminates in (S2), then $B$ is an optimal basis for (1). If termination occurs in (S4), then (1) is unbounded. Otherwise, the $B$ and $X_{B}$ produced in (S5) satisfy the input conditions, and the corresponding objective-function value is no bigger than for the initial $X$.

Proof. (Optimality) By assumption, $B$ is feasible. Suppose termination occurs in (S2). Let $d=c-A^{T} \pi$, where $\pi$ is as computed in (S1). Now for any feasible $x$, $d^{T} x=c^{T} x-\pi^{T} A x=c^{T} x-\pi^{T} b$. Thus, minimizing $d^{T} x$ is equivalent to minimizing $c^{T} x$. But for $x$ feasible, one has

$$
\begin{aligned}
d^{T} x & =d_{B}^{T} x_{B}+d_{N_{l}}^{T} x_{N_{l}}+d_{N_{u}}^{T} x_{N_{u}}+d_{N_{f r}}^{T} x_{N_{f r}}+d_{N_{f x}}^{T} x_{N_{f x}} \\
& =d_{B}^{T} X_{B}+d_{N_{l}}^{T} x_{N_{l}}+d_{N_{u}}^{T} x_{N_{u}}+d_{N_{f r}}^{T} X_{N_{f r}}+d_{N_{f x}}^{T} X_{N_{f x}} \\
& \geq d_{B}^{T} X_{B}+d_{N_{l}}^{T} l_{N_{l}}+d_{N_{u}}^{T} u_{N_{u}}+d_{N_{f r}}^{T} X_{N_{f r}}+d_{N_{f x}}^{T} X_{N_{f x}} \\
& =d^{T} X .
\end{aligned}
$$

The second equality follows because $d_{B}=0, d_{N_{f r}}=0$ and $X_{N_{f x}}=x_{N_{f x}}$. The inequality follows because $d_{N_{l}} \geq 0, x_{N_{l}} \geq l_{N_{l}}, d_{N_{u}} \leq 0$ and $x_{N_{u}} \leq u_{N_{u}}$. Hence, $B$ is optimal.

(Unboundedness) Suppose that $d_{j_{e}}<0$, and for $\alpha \geq 0$ define

$$
\begin{aligned}
X_{j_{e}}^{\alpha} & =X_{j_{e}}+\alpha \\
X_{B}^{\alpha} & =X_{B}-\alpha y, \text { and } \\
X_{j}^{\alpha} & =X_{j} \text { for } j \notin B \cup\left\{j_{e}\right\}
\end{aligned}
$$

where $\boldsymbol{B} y=A_{j_{e}}$, and $X$ is given by (2). It is straightforward to see that $A X^{\alpha}=b$. Suppose that $\alpha \leq \Theta$. Then clearly $l_{j_{e}} \leq X_{j_{e}}^{\alpha} \leq u_{j_{e}}$, and so $l_{N} \leq X_{N}^{\alpha} \leq u_{N}$; moreover, $0 \leq \alpha \leq \min _{i} \Theta_{i}$ implies $l_{B} \leq X_{B}^{\alpha} \leq u_{B}$. Hence, $X^{\alpha}$ is feasible, and

$$
\begin{aligned}
c^{T} X^{\alpha} & =c^{T} X+\alpha c_{j_{e}}-\alpha c_{B}^{T} y \\
& =c^{T} X+\alpha c_{j_{e}}-\alpha \pi^{T} A_{j_{e}} \\
& =c^{T} X+\alpha d_{j_{e}}
\end{aligned}
$$

It follow that if $\Theta=+\infty$, then (1) is unbounded. A similar argument applies when $d_{j_{e}}>0$, taking $X_{j_{e}}^{\alpha}=X_{j_{e}}-\alpha$ and $X_{B}^{\alpha}=X_{B}+\alpha y$. 
(Update) Finally, it must be shown that if neither optimality nor unboundedness occurs, then the new $B$ is a feasible basis with corresponding values given by the new $X_{B}$. Note that the new $X_{B}$ is nothing but $X_{B}^{\alpha}$, where $\alpha=\Theta$ and $X^{\alpha}$ is as defined in the unboundedness proof. Hence, the new $X_{B}$ does give the vector of values of the basic variables, if the new $B$ is a basis; moreover, $c^{T} X^{\Theta} \leq c^{T} X$ by the choices of $d_{j_{e}}$ and $\Theta$.

If (S5.1) occurs, the new $B$ is the old $B$, and so obviously a basis. For the remaining case, it must be shown that the new $\boldsymbol{B}$ is nonsingular. Denoting the new $\boldsymbol{B}$ by $\bar{B}$, it follows that

$$
\bar{B}=\boldsymbol{B} E
$$

where

$$
E=I+\left(y-e_{i_{l}}\right) e_{i_{l}}^{T},
$$

$I$ is an $m \times m$ identity, and $e_{i_{l}}$ is a unit vector with a 1 in row $i_{l}$. But $\boldsymbol{B}$ is nonsingular, so $B E v=0$ implies

$$
v+v_{i_{l}}\left(y-e_{i_{l}}\right)=E v=0,
$$

and hence that $v=0$, since $y_{i_{l}} \neq 0$ by the definition of $\Theta$. It follows that $\overline{\boldsymbol{B}}$ is nonsingular.

\section{The Netlib Test Problems}

The computational tests reported here make use of the netlib problems. ${ }^{3}$ To the knowledge of this author, the netlib set does not contain any randomly generated problems. All are at least real in the sense that they were humanly generated and represent "real attempts" at modeling.

Some general comments on netlib are probably in order. First, many of the problems in the set are there because they have caused difficulties for the simplex method. Such problems are useful for the purpose of developing robust code, but may not be the best for testing speed and choosing default parameter settings. Second, there are several classes of problems on the list. Two examples are pilot (pilot4, perold, pilotwe, pilotnov, pilotja, pilots and pilot87) and fit (fit1p, fit1d, fit2p and fit2d). These classes do help illustrate how performance changes as problem size increases; however, in practice it does not make much sense to run all the problems in such a class without first doing some testing, based on smaller instances in the class. For example, the ' $p$ ' and 'd' versions from the fit class represent equivalent, dual formulations of the same problem. After having solved fitlp and fitld, it is obvious that the ' $\mathrm{d}$ ' formulation is much easier for the simplex method. From a purely practical viewpoint, there is then little reason to consider solving the fit2p. Similarly, it is well known that the pilot problems are "poorly scaled." The solution

\footnotetext{
${ }^{3}$ Copies of these problems, assembled by D. M. Gay, can be obtained (at this writing) by "anonymous ftp" to research.att.com (userid: anonymous, password: $\langle b l a n k\rangle$ ). The actual data is contained in the directory dist/lpdata.
} 
times on all these problems can be easily improved by using a more aggressive scaling approach. ${ }^{4}$

Three tables of netlib data are given at the end of this section (Tables I, II and III). Table I contains problem data and Tables II and III numerical information on optimal bases, as computed by CPLEX. Blanks indicate zeros. Problems are ordered by the number of constraint nonzeros. For "pictures" of the individual netlib problems see [7] or [8]. (The technical report [7] contains significantly more data than the published version.)

In Table I, counts are given for the various row (constraint) and column (variable) types. Boxed variables are variables $x_{j}$ with bounds of the form $l_{j} \leq x_{j} \leq u_{j}$ where both $l_{j}$ and $u_{j}$ are finite. Fixed variables are just that, fixed $\left(l_{j}=u_{j}\right)$. As one might expect, nonnegative variables dominate.

In addition to the standard row types, ranged and free rows are listed. Ranged rows correspond to constraints of the form

$$
\underline{b}_{i} \leq a_{i}^{T} x \leq \bar{b}_{i}
$$

and are modeled by introducing a variable, say $y_{i}$, with appropriate bounds, and using this variable to replace the above pair of constraints with a single equality constraint:

$$
\begin{aligned}
& a_{i}^{T} x-y=0 \\
& \underline{b}_{i} \leq y_{i} \leq \bar{b}_{i}
\end{aligned}
$$

Free or unconstrained rows are typically added by users as accounting devices or as a way of encoding alternate objective functions. They are ignored during optimization.

One of the first considerations encountered in implementing virtually any numerical algorithm is the setting of tolerances, frequently replacements for some of the 0's that occur in tests in the mathematical description. (A careful discussion of tolerance settings is beyond the scope of the present paper.) Two such tolerances for the simplex method are the feasibility and optimality tolerances, used, respectively, to determine whether basic variables satisfy their bounds and whether the vector $d$ of reduced costs satisfies the optimality conditions given in Step S2. The default CPLEX values for the feasibility and optimality tolerances are 1.0E-6 (0.000001); these tolerances can be independently reset to values as low as $1.0 \mathrm{E}-9$.

A third important tolerance is the "pivot threshold" used in computing an LUfactorization of $\boldsymbol{B}$. The factorization algorithm used in CPLEX is based on the method of Markowitz [10] and is implemented as described in [12]. In this scheme, the elimination process is carried out using a relaxation of partial pivoting [4] called threshold pivoting. The threshold is a number $0<\alpha \leq 1$. A pivot element is considered acceptable if it is at least $\alpha$ times the maximum absolute value of a nonzero in the column or row being eliminated. CPLEX takes as the default value $\alpha=0.01$;

\footnotetext{
${ }^{4}$ The default scaling used by CPLEX is very simple. First, every row is divided by the maximum absolute value in that row. When that step is complete, every column is divided by the maximum absolute value in that column.
} 
this tolerance can be reset to any value between 0.0001 and 0.99999 . (Setting $\alpha=1.0$ is not allowed for numerical reasons.)

To obtain objective values for Tables II and III that were as accurate as possible, all problems were first solved using default settings and then reoptimized from that point with optimality and feasibility tolerances set to $1.0 \mathrm{E}-9$ and threshold $\alpha=$ 0.99999. Computations were carried out on a SPARCstation 2 using IEEE floatingpoint arithmetic (implying between 15 and 17 digits of accuracy). Data are given for both the solution obtained with defaults and the solution after reoptimization with the indicated "tighter" settings. Data are also presented for both scaled and unscaled versions of the optimal bases. However, it should be noted that no problems were actually solved in unscaled form. CPLEX does not permit that.

The condition numbers [4] reported in Table II were computed in the $L_{\infty}$-norm using an LU-factorization of the reoptimized optimal basis. The relative objective error is given by the formula $\left|\left(z_{d}-z_{\tau}\right) / z_{\tau}\right|$, where $z_{d}$ is the objective value obtained running defaults and $z_{r}$ is the value after reoptimization. The "Default Settings Objective Value" column in Table II is identical to the first 11 digits of the "Objective Value" column in Table I.

Residuals reported are maximum absolute deviations: $\|A X-b\|_{\infty}$ and $\| c_{B}^{T}-$ $\pi^{T} \boldsymbol{B} \|_{\infty}$, where $X$ is as defined in (2), and the system $\pi^{T} \boldsymbol{B}=c_{B}^{T}$ is taken from Step S1 of Algorithm 2.2. The entries in the bound and reduced-cost columns were computed as follows:

bound residual $=\max \left\{\max \left\{X_{j}-u_{j}, l_{j}-X_{j}, 0\right\}: j \in B\right\}$, and reduced-cost residual $=\max \left\{\Delta_{l}, \Delta_{u}, \Delta_{f}\right\}$,

where

$$
\begin{aligned}
\Delta_{l} & =\max \left\{\max \left\{c_{j}-\pi^{T} A_{j}, 0\right\}: j \in N_{l}\right\}, \\
\Delta_{u} & =\max \left\{\max \left\{\pi^{T} A_{j}-c_{j}, 0\right\}: j \in N_{u}\right\}, \text { and } \\
\Delta_{f} & =\max \left\{\left|c_{j}-\pi^{T} A_{j}\right|: j \in N_{f r}\right\} .
\end{aligned}
$$

and the expressions for $\Delta_{l}$ and $\Delta_{u}$ use the fact that all netlib problems have been formulated as minimization problems. As previously indicated, the default tolerances for the bound and reduced-cost residuals are 1.0E-6. Residuals must in each case be no bigger than the corresponding tolerance for the solution to be designated as optimal. 
TABLE I

Netlib Problem Data

\begin{tabular}{|c|c|c|c|c|c|c|c|c|c|c|c|c|c|c|c|c|}
\hline \multirow{2}{*}{\multicolumn{2}{|c|}{ PROBLEM }} & \multirow{2}{*}{$\begin{array}{c}\text { Optimal } \\
\text { Value }\end{array}$} & \multirow[b]{2}{*}{ Cons. } & \multirow[b]{2}{*}{ Vars. [ } & \multicolumn{3}{|c|}{ Nonzeros } & \multicolumn{4}{|c|}{ Constraint Types } & \multicolumn{5}{|c|}{ Variable Types } \\
\hline & & & & & Cons. & Obj. & RHS & Less & Greater & Equal & Range & Nonneg. & Box 1 & Free & Fix & Other \\
\hline 1 & afiro & $-4.6475314286 \mathrm{E}+02$ & 27 & 32 & 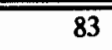 & 5 & 7 & $\overline{19}$ & & 8 & & 32 & & & & \\
\hline 2 & sc50b & $-7.0000000000 E+01$ & 50 & 48 & 118 & 1 & 5 & 30 & & 20 & & 48 & & & & \\
\hline 3 & sc50a & $-6.4575077059 E+01$ & 50 & 48 & 130 & 1 & 10 & 30 & & 20 & & 48 & & & & \\
\hline 4 & sc105 & $-5.2202061212 E+01$ & 105 & 103 & 280 & 1 & 20 & 60 & & 45 & & 103 & & & & \\
\hline 5 & kb2 & $-1.7499001299 E+03$ & 43 & 41 & 286 & 5 & 0 & 12 & 15 & 16 & & 32 & 9 & & & \\
\hline 6 & adlittle & $2.2549496316 \mathrm{E}+05$ & 56 & 97 & 383 & 82 & 37 & 40 & 1 & 15 & & 97 & & & & \\
\hline 7 & scagr 7 & $-2.3313898243 E+06$ & 129 & 140 & 420 & 133 & 53 & 38 & 7 & 84 & & 140 & & & & \\
\hline 8 & stocfor1 & $-4.1131976219 E+04$ & 117 & 111 & 447 & 27 & 8 & 48 & 6 & 63 & & 111 & & & & \\
\hline$P$ & blend & $-3.0812149846 \mathrm{E}+01$ & 74 & 83 & 491 & 30 & 8 & 31 & & 43 & & 83 & & & & \\
\hline 10 & sc205 & $-5.2202061212 \mathrm{E}+01$ & 205 & 203 & 551 & 1 & 38 & 114 & & 91 & & 203 & & & & \\
\hline 11 & recipe & $-2.6661600000 \mathrm{E}+02$ & 91 & 180 & 663 & 89 & 0 & $\overline{6}$ & 18 & 67 & & 85 & 69 & & 26 & \\
\hline 12 & share2b & $-4.1573224074 \mathrm{E}+02$ & 96 & 79 & 694 & 36 & 24 & 83 & & 13 & & 79 & & & & \\
\hline 13 & vtpbase & $1.2983146246 \mathrm{E}+05$ & 198 & 203 & 908 & 6 & 59 & 133 & 10 & 55 & & 87 & 65 & 1 & 18 & 32 \\
\hline 14 & lotfi & $-2.5264706062 \mathrm{E}+01$ & 153 & 308 & 1078 & 8 & 49 & 42 & 16 & 95 & & 308 & & & & \\
\hline 15 & sharelb & $-7.6589318579 E+04$ & 117 & 225 & 1151 & 31 & 103 & 28 & & 89 & & 225 & & & & \\
\hline 16 & boeing2 & $-3.1501872802 \mathrm{E}+02$ & 166 & 143 & 1196 & 143 & 39 & 1 & 142 & 23 & 19 & 89 & 54 & & & \\
\hline 17 & scorpion & $1.8781248227 \mathrm{E}+03$ & 388 & 358 & 1426 & 282 & 76 & 48 & 60 & 280 & & 358 & & & & \\
\hline 18 & bore3d & $1.3730803942 \mathrm{E}+03$ & 233 & 315 & 1429 & 96 & 0 & 19 & & 214 & & 302 & 11 & & 1 & 1 \\
\hline 19 & scagr 25 & $-1.4753433061 \mathrm{E}+07$ & 471 & 500 & 1554 & 475 & 179 & 146 & 25 & 300 & & 500 & & & & \\
\hline 20 & sctap1 & $1.4122500000 \mathrm{E}+03$ & 300 & 480 & 1692 & 360 & 154 & & 180 & 120 & & 480 & & & & \\
\hline 21 & capri & $2.6900129138 \mathrm{E}+03$ & 271 & 353 & 1767 & 19 & 130 & 75 & 54 & 142 & & 192 & 131 & 14 & 16 & \\
\hline 22 & brandy & $1.5185098965 \mathrm{E}+03$ & 220 & 249 & 2148 & 2 & 54 & 54 & & 166 & & 249 & & & & \\
\hline 23 & israel & $-8.9664482186 \mathrm{E}+05$ & 174 & 142 & 2269 & 89 & 171 & 174 & & & & 142 & & & & \\
\hline 24 & finnis & $1.7279106560 \mathrm{E}+05$ & 497 & 614 & 2310 & 404 & 116 & 302 & 148 & 47 & & 492 & 36 & & 45 & 41 \\
\hline 25 & gfrdpnc & $6.9022359995 E+06$ & 616 & 1092 & 2377 & 1090 & 68 & 68 & & 548 & & 834 & 258 & & & \\
\hline 26 & $\operatorname{scsd} 1$ & $8.6666666743 \mathrm{E}+00$ & 77 & 760 & 2388 & 760 & 1 & & & 77 & & 760 & & & & \\
\hline 27 & etamacro & $-7.5571523337 \mathrm{E}+02$ & 400 & 688 & 2409 & 80 & 24 & 48 & 80 & 272 & & 426 & 135 & & 82 & 45 \\
\hline 28 & agg & $-3.5991767287 \mathrm{E}+07$ & 488 & 163 & 2410 & 131 & 432 & 405 & 47 & 36 & & 163 & & & & \\
\hline 29 & bandm & $-1.5862801845 E+02$ & 305 & 472 & 2494 & 165 & 118 & & & 305 & & 472 & & & & \\
\hline 30 & e226 & $-1.8751929066 \mathrm{E}+01$ & 223 & 282 & 2578 & 189 & 99 & 185 & 5 & 33 & & 282 & & & & \\
\hline 31 & scfxml & $1.8416759028 \mathrm{E}+04$ & 330 & 457 & 2589 & 23 & 116 & 143 & & 187 & & 457 & & & & \\
\hline 32 & grow7 & $-4.7787811815 E+07$ & 140 & 301 & 2612 & 21 & 0 & & & 140 & & 21 & 280 & & & \\
\hline 33 & standata & $1.2576995000 \mathrm{E}+03$ & 359 & 1183 & 3031 & 7 & 7 & 199 & & 160 & & 1063 & 104 & & 16 & \\
\hline 34 & scrs8 & $9.0429695380 \mathrm{E}+02$ & 490 & 1169 & 3182 & 847 & 77 & 59 & 47 & 384 & & 1169 & & & & \\
\hline 35 & beaconfd & $3.3592485807 \mathrm{E}+04$ & 173 & 262 & 3375 & 101 & 67 & 33 & & 140 & & 262 & & & & \\
\hline 36 & boeing1 & $-3.3521356751 E+02$ & 351 & 384 & 3485 & 380 & 146 & 4 & 249 & 9 & 89 & 228 & 156 & & & \\
\hline 37 & shell & $1.2088253460 \mathrm{E}+09$ & 536 & 1775 & 3556 & 1344 & 2 & 2 & & 534 & & 1399 & 117 & & 250 & 9 \\
\hline 38 & standmps & $1.4060175000 \mathrm{E}+03$ & 467 & 1075 & 3679 & 7 & 115 & 199 & & 268 & & 955 & 104 & & 16 & \\
\hline 39 & stair & $-2.5126695119 E+02$ & 356 & 467 & 3856 & 1 & 70 & 147 & & 209 & & 373 & 6 & 6 & 82 & \\
\hline 40 & degen 2 & $-1.4351780000 \mathrm{E}+03$ & 444 & 534 & 3978 & 471 & 243 & 223 & & 221 & & 534 & & & & \\
\hline 41 & agg2 & $-2.0239252356 \mathrm{E}+07$ & 516 & 302 & 4284 & 231 & 472 & 456 & & 60 & & 302 & & & & \\
\hline 42 & agg3 & $1.0312115935 \mathrm{E}+07$ & 516 & 302 & 4300 & 231 & 467 & 456 & & 60 & & 302 & & & & \\
\hline 43 & sesd6 & $5.0500000077 \mathrm{E}+01$ & 147 & 1350 & 4316 & 1350 & 9 & & & 147 & & 1350 & & & & \\
\hline 44 & ship04s & $1.7987147004 \mathrm{E}+06$ & 402 & 1458 & 4352 & 1458 & 263 & 40 & 8 & 354 & & 1458 & & & & \\
\hline 45 & seba & $1.5711600000 \mathrm{E}+04$ & 515 & 1028 & 4352 & 522 & 9 & & 1 & 507 & 7 & 521 & 507 & & & \\
\hline 46 & tuff & $2.9214776509 \mathrm{E}-01$ & 333 & 587 & 4520 & 3 & 0 & 15 & 26 & 292 & & 559 & 26 & 2 & & \\
\hline 47 & forplan & $-6.6421896127 \mathrm{E}+02$ & 161 & 420 & 4563 & 353 & 15 & 50 & 20 & 90 & 1 & 396 & 21 & & 3 & \\
\hline 48 & bnl1 & $1.9776295615 \mathrm{E}+03$ & 643 & 1175 & 5121 & 1008 & 276 & 205 & 206 & 232 & & 1175 & & & & \\
\hline 49 & pilot4 & $-2.5811392589 \mathrm{E}+03$ & 410 & 1000 & 5141 & 4 & 165 & 26 & 97 & 287 & & 635 & 247 & 88 & 30 & \\
\hline 50 & scfxm2 & $3.6660261565 \mathrm{E}+04$ & 660 & 914 & 5183 & 46 & 237 & 286 & & 374 & & 914 & & & & \\
\hline
\end{tabular}


TABLE I

Netlib Problem Data

\begin{tabular}{|c|c|c|c|c|c|c|c|c|c|c|c|c|c|c|c|c|}
\hline \multirow{2}{*}{\multicolumn{2}{|c|}{ PROBLEM }} & \multirow{2}{*}{$\begin{array}{l}\text { Optimal } \\
\text { Value }\end{array}$} & \multirow[b]{2}{*}{ Cons. } & \multirow[b]{2}{*}{ Vars. } & \multicolumn{3}{|c|}{ Nonzeros } & \multicolumn{4}{|c|}{ Constraint Types } & \multicolumn{5}{|c|}{ Variable Types } \\
\hline & & & & & Cons. & Obj. & RHS & Less & Greater & Equal & Range & Nonneg. & Box & Free & Fix & Other \\
\hline 51 & grow15 & $-1.0687094129 \mathrm{E}+08$ & 300 & 645 & 5620 & 45 & 0 & & & 300 & & 45 & 600 & & & \\
\hline 52 & perold & $-9.3807552782 E+03$ & 625 & 1376 & 6018 & 8 & 215 & 40 & 90 & 495 & & 951 & 266 & 88 & 64 & 7 \\
\hline 53 & ffffrf 800 & $5.5567956482 E+05$ & 524 & 854 & 6227 & 8 & 204 & 93 & 81 & 350 & & 854 & & & & \\
\hline 54 & ship04l & $1.7933245380 \mathrm{E}+06$ & 402 & 2118 & 6332 & 2118 & 263 & 40 & 8 & 354 & & 2118 & & & & \\
\hline 55 & sctap2 & $1.7248071429 E+03$ & 1090 & 1880 & 6714 & 1410 & 521 & & 620 & 470 & & 1880 & & & & \\
\hline 56 & ganges & $-1.0958573613 E+05$ & 1309 & 1681 & 6912 & 109 & 491 & 25 & & 1284 & & 1277 & 397 & & & 7 \\
\hline 57 & ship08s & $1.9200982105 E+06$ & 778 & 2387 & 7114 & 2387 & 413 & 72 & 8 & 698 & & 2387 & & & & \\
\hline 58 & sierra & $1.5394362184 E+07$ & 1227 & 2036 & 7302 & 1950 & 781 & 633 & 66 & 528 & & & 2016 & & 20 & \\
\hline 59 & $\operatorname{scf} x \mathrm{~m} 3$ & $5.4901254550 \mathrm{E}+04$ & 990 & 1371 & 7777 & 69 & 358 & 429 & & 561 & & 1371 & & & & \\
\hline 60 & ship12s & $1.4892361344 \mathrm{E}+06$ & 1151 & 2763 & 8178 & 2763 & 653 & 101 & 5 & 1045 & & 2763 & & & & \\
\hline 61 & grow22 & $-1.6083433648 E+08$ & 440 & 946 & 8252 & 66 & 0 & & & 440 & & 66 & 880 & & & \\
\hline 62 & stocfor2 & $-3.9024408538 E+04$ & 2157 & 2031 & 8343 & 1149 & 8 & 888 & 126 & 1143 & & 2031 & & & & \\
\hline 63 & $\operatorname{scsd8}$ & $9.0499999993 \mathrm{E}+02$ & 397 & 2750 & 8584 & 2750 & 15 & & & 397 & & 2750 & & & & \\
\hline 64 & sctap3 & $1.4240000000 E+03$ & 1480 & 2480 & 8874 & 1860 & 682 & & 860 & 620 & & 2480 & & & & \\
\hline 65 & pilotwe & $-2.7201075328 \mathrm{E}+06$ & 722 & 2789 & 9126 & 92 & 73 & 30 & 109 & 583 & & 2335 & 294 & 80 & 78 & 2 \\
\hline 66 & maros & $-5.8063743701 E+04$ & 846 & 1443 & 9614 & 392 & 42 & 399 & 124 & 323 & & 1405 & & & 35 & 3 \\
\hline 67 & fitlp & $9.1463780924 E+03$ & 627 & 1677 & 9868 & 1026 & 627 & & & 627 & & 1278 & 399 & & & \\
\hline 68 & 25 fv47 & $5.5018458883 E+03$ & 821 & 1571 & 10400 & 727 & 287 & 305 & & 516 & & 1571 & & & & \\
\hline 69 & czprob & $2.1851966989 E+06$ & 929 & 3523 & 10669 & 3504 & 860 & 38 & 1 & 890 & & 3294 & & & 229 & \\
\hline 70 & ship081 & $1.9090552114 \mathrm{E}+06$ & 778 & 4283 & 12802 & 4283 & 413 & 72 & 8 & 698 & & 4283 & & & & \\
\hline 71 & pilotnov & $-4.4972761882 E+03$ & 975 & 2172 & 13057 & 72 & 332 & 151 & 123 & 701 & & 1628 & 340 & & 204 & \\
\hline 72 & nesm & $1.4076036488 \mathrm{E}+07$ & 662 & 2923 & 13288 & 700 & 542 & 94 & & 480 & 88 & 1009 & 1508 & & 175 & 231 \\
\hline 73 & fit1d & $-9.1463780924 \mathrm{E}+03$ & 24 & 1026 & 13404 & 1026 & 0 & 12 & 11 & 1 & & & 1026 & & & \\
\hline 74 & bnl2 & $1.8112365404 E+03$ & 2324 & 3489 & 13999 & 2125 & 441 & 482 & 515 & 1327 & & 3489 & & & & \\
\hline 75 & pilotja & $-6.1131364654 E+03$ & 940 & 1988 & 14698 & 8 & 309 & 151 & 128 & 661 & & 1250 & 339 & 88 & 311 & \\
\hline 76 & ship121 & $1.4701879193 \mathrm{E}+06$ & 1151 & 5427 & 16170 & 5427 & 653 & 101 & 5 & 1045 & & 5427 & & & & \\
\hline 77 & cycle & $-5.2263930249 E+00$ & 1903 & 2857 & 20720 & 602 & 0 & 146 & 368 & 1389 & & 2773 & 77 & 7 & & \\
\hline 78 & $80 \mathrm{bau} 3 \mathrm{~b}$ & $9.8722419241 \mathrm{E}+05$ & 2262 & 9799 & 21002 & 8061 & 346 & 35 & 2227 & & & 6244 & 2986 & & 498 & 71 \\
\hline 79 & degen 3 & $-9.8729400000 \mathrm{E}+02$ & 1503 & 1818 & 24646 & 1584 & 594 & 786 & & 717 & & 1818 & & & & \\
\hline 80 & truss & $4.5881584719 E+05$ & 1000 & 8806 & 27836 & & & & & 1000 & & 8806 & & & & \\
\hline 81 & greenbea & $-7.2555248130 \mathrm{E}+07$ & 2392 & 5405 & 30877 & 622 & 0 & 107 & 86 & 2199 & & 4996 & 290 & & 103 & 16 \\
\hline 82 & greenbeb & $-4.3022602612 E+06$ & 2392 & 5405 & 30877 & 622 & 0 & 107 & 86 & 2199 & & 4978 & 291 & 4 & 115 & 17 \\
\hline 83 & $d 2 q 06 c$ & $1.2278421081 \mathrm{E}+05$ & 2171 & 5167 & 32417 & 3257 & 874 & 664 & & 1507 & & 5167 & & & & \\
\hline 84 & woodw & $1.3044763331 E+00$ & 1098 & 8405 & 37474 & 4 & 34 & 9 & 4 & 1085 & & 8405 & & & & \\
\hline 85 & pilots & $-5.5748972928 \mathrm{E}+02$ & 1441 & 3652 & 43167 & 53 & 282 & 1191 & 17 & 233 & & 2320 & 1040 & & 203 & 89 \\
\hline 86 & fit $2 p$ & $6.8464293294 E+04$ & 3000 & 13525 & 50284 & 10500 & 1500 & & & 3000 & & 6025 & 7500 & & & \\
\hline 87 & stocfor 3 & $-3.9976783944 E+04$ & 16675 & 15695 & 64875 & 9129 & 8 & 6866 & 980 & 8829 & & 15695 & & & & \\
\hline 88 & woodlp & $1.4429024116 \mathrm{E}+00$ & 244 & 2594 & 70215 & 1 & 2 & & 1 & 243 & & 2594 & & & & \\
\hline 89 & pilot87 & $3.0171034733 \mathrm{E}+02$ & 2030 & 4883 & 73152 & 652 & 274 & 1708 & 89 & 233 & & 2971 & 1578 & & 220 & 114 \\
\hline 90 & fit $2 d$ & $-6.8464293294 E+04$ & 25 & 10500 & 129018 & 9000 & 0 & 10 & 14 & 1 & & & 10500 & & & \\
\hline
\end{tabular}


TABLE II

Stability of Optimal Bases

\begin{tabular}{|c|c|c|c|c|c|c|}
\hline \multicolumn{2}{|c|}{ PROBLEM } & \multicolumn{2}{|c|}{$\begin{array}{l}\text { Reoptimized Basis } \\
\text { Condition Number }\end{array}$} & $\begin{array}{c}\text { Default Settings } \\
\text { Objective } \\
\text { Value } \\
\end{array}$ & $\begin{array}{c}\text { Reoptimized } \\
\text { Objective } \\
\text { Value } \\
\end{array}$ & $\begin{array}{c}\text { Relative } \\
\text { Objective } \\
\text { Error } \\
\end{array}$ \\
\hline$\overline{1}$ & afiro & $3.21 \mathrm{E}+01$ & $3.13 \mathrm{E}+01$ & $-4.647531428571 \mathrm{E}+02$ & $-4.647531428571 \mathrm{E}+02$ & \\
\hline 2 & sc50b & $1.23 \mathrm{E}+02$ & $1.23 E+02$ & $-7.000000000000 \mathrm{E}+01$ & $-7.000000000000 \mathrm{E}+01$ & \\
\hline 3 & $\operatorname{sc} 50 \mathrm{a}$ & $1.28 \mathrm{E}+02$ & $9.52 \mathrm{E}+01$ & $-6.457507705856 E+01$ & $-6.457507705856 E+01$ & \\
\hline 4 & $\operatorname{sc1} 105$ & $4.83 \mathrm{E}+02$ & $3.28 \mathrm{E}+02$ & $-5.220206121171 E+01$ & $-5.220206121171 E+01$ & \\
\hline 5 & kb2 & $1.56 \mathrm{E}+06$ & $2.87 \mathrm{E}+03$ & $-1.749900129906 \mathrm{E}+03$ & $-1.749900129906 \mathrm{E}+03$ & \\
\hline 6 & adlittle & $5.47 \mathrm{E}+03$ & $4.35 \mathrm{E}+02$ & $2.254949631624 \mathrm{E}+05$ & $2.254949631624 \mathrm{E}+05$ & \\
\hline 7 & scagr7 & $1.04 \mathrm{E}+04$ & $4.24 E+03$ & $-2.331389824331 E+06$ & $-2.331389824331 E+06$ & \\
\hline 8 & stocforl & $5.07 \mathrm{E}+05$ & $1.93 E+02$ & $-4.113197621944 \mathrm{E}+04$ & $-4.113197621944 \mathrm{E}+04$ & \\
\hline 9 & blend & $1.33 E+04$ & $4.07 \mathrm{E}+02$ & $-3.081214984583 \mathrm{E}+01$ & $-3.081214984583 E+01$ & \\
\hline 10 & $\mathrm{sc} 205$ & $2.30 \mathrm{E}+03$ & $1.75 E+03$ & $-5.220206121171 \mathrm{E}+01$ & $-5.220206121171 \mathrm{E}+01$ & \\
\hline 11 & recipe & $4.82 \mathrm{E}+05$ & $1.04 E+02$ & $-2,666160000000 \mathrm{E}+02$ & $-2.666160000000 \mathrm{E}+02$ & \\
\hline 12 & share2b & $1.29 E+06$ & $7.49 \mathrm{E}+03$ & $-4.157322407414 \mathrm{E}+02$ & $-4.157322407414 E+02$ & \\
\hline 13 & vtpbase & $1.85 \mathrm{E}+09$ & $9.23 \mathrm{E}+04$ & $1.298314624614 \mathrm{E}+05$ & $1.298314624614 E+05$ & \\
\hline 14 & lotfi & $1.05 \mathrm{E}+07$ & $3.76 \mathrm{E}+04$ & $-2.526470606188 E+01$ & $-2.526470606188 \mathrm{E}+01$ & \\
\hline 15 & sharelb & $2.39 \mathrm{E}+07$ & $3.97 \mathrm{E}+03$ & $-7.658931857919 E+04$ & $-7.658931857919 \mathrm{E}+04$ & \\
\hline 16 & boeing2 & $4.89 \mathrm{E}+06$ & $5.34 E+04$ & $-3.150187280152 \mathrm{E}+02$ & $-3.150187280152 E+02$ & \\
\hline 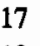 & scorpion & $3.24 E+03$ & $2.38 \mathrm{E}+03$ & $1.878124822738 \mathrm{E}+03$ & $1.878124822738 E+03$ & \\
\hline 18 & bore3d & $1.54 \mathrm{E}+07$ & $6.43 E+04$ & $1.373080394208 \mathrm{E}+03$ & $1.373080394208 \mathrm{E}+03$ & \\
\hline 19 & scagr25 & $1.38 \mathrm{E}+04$ & $4.18 \mathrm{E}+03$ & $-1.475343306077 \mathrm{E}+07$ & $-1.475343306077 \mathrm{E}+07$ & \\
\hline 20 & sctap1 & $6.82 \mathrm{E}+04$ & $1.68 \mathrm{E}+02$ & $1.412250000000 \mathrm{E}+03$ & $1.412250000000 \mathrm{E}+03$ & \\
\hline 21 & capri & $1.13 E+06$ & $3.84 \mathrm{E}+04$ & $2.690012913768 \mathrm{E}+03$ & $13768 \mathrm{E}+03$ & \\
\hline 22 & brandy & $1.24 E+05$ & $1.32 \mathrm{E}+03$ & $1.518509896488 \mathrm{E}+03$ & $1.518509896488 \mathrm{E}+03$ & \\
\hline 23 & israel & $1.53 E+07$ & $2.66 \mathrm{E}+04$ & $-8.966448218630 \mathrm{E}+05$ & $-8.966448218630 \mathrm{E}+05$ & \\
\hline 24 & finnis & $9.91 E+04$ & $3.66 \mathrm{E}+03$ & $1.727910655956 \mathrm{E}+05$ & $1.727910655956 \mathrm{E}+05$ & \\
\hline ; & gfrdpnc & $1.94 \mathrm{E}+05$ & $1.38 \mathrm{E}+05$ & $6.902235999549 \mathrm{E}+06$ & $6.902235999549 \mathrm{E}+06$ & \\
\hline 26 & $\operatorname{sesd} 1$ & $7.65 E+01$ & $7.65 E+01$ & $8.6666666674333 E+\infty 0$ & $8.666666674333 \mathrm{E}+00$ & \\
\hline 27 & etamacro & $5.53 \mathrm{E}+06$ & $5.66 \mathrm{E}+03$ & $-7.557152298914 E+02$ & $-7.557152333749 \mathrm{E}+02$ & 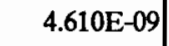 \\
\hline 28 & agg & $5.08 \mathrm{E}+07$ & $2.04 E+05$ & $-3.599176728658 E+07$ & $-3.599176728658 E+07$ & \\
\hline 29 & bandm & $2.39 E+05$ & $5.16 E+03$ & $-1.586280184501 \mathrm{E}+02$ & $-1.586280184501 E+02$ & \\
\hline 30 & e226 & $1.57 \mathrm{E}+07$ & $1.11 \mathrm{E}+04$ & $-1.875192906637 \mathrm{E}+01$ & $-1.875192906637 \mathrm{E}+01$ & \\
\hline 31 & scfxm1 & $2.88 \mathrm{E}+06$ & $3.93 \mathrm{E}+03$ & $1.841675902835 \mathrm{E}+04$ & $1.841675902835 \mathrm{E}+04$ & \\
\hline 32 & grow 7 & $4.16 E+02$ & $3.64 \mathrm{E}+02$ & $-4.778781181471 E+07$ & $-4.778781181471 E+07$ & \\
\hline 33 & standata & $3.23 \mathrm{E}+04$ & $8.53 E+02$ & $1.257699500000 \mathrm{E}+03$ & $1.257699500000 \mathrm{E}+03$ & \\
\hline 34 & scrs8 & $5.65 E+05$ & $2.78 \mathrm{E}+03$ & $9.042969538008 E+02$ & $9.042969538008 \mathrm{E}+02$ & \\
\hline 35 & beaconfd & $1.04 E+04$ & $4.02 \mathrm{E}+01$ & $3.359248580720 \mathrm{E}+04$ & $3.359248580720 \mathrm{E}+04$ & \\
\hline 36 & boeing1 & $5.86 \mathrm{E}+07$ & $2.29 \mathrm{E}+05$ & $-3.352135675071 E+02$ & $-3.352135675071 \mathrm{E}+02$ & \\
\hline 37 & shell & $1.20 \mathrm{E}+02$ & $1.20 \mathrm{E}+02$ & $1.208825346000 \mathrm{E}+09$ & $1.208825346000 \mathrm{E}+09$ & \\
\hline 38 & standn & $3.28 \mathrm{E}+04$ & $2.73 E+03$ & $1.406017500000 \mathrm{E}+03$ & $1.406017500000 \mathrm{E}+03$ & \\
\hline 39 & stair & $8.94 E+04$ & $2.16 \mathrm{E}+04$ & $-2.512669511930 \mathrm{E}+02$ & $-2.512669511930 \mathrm{E}+02$ & \\
\hline 40 & degen 2 & $3.80 \mathrm{E}+03$ & $3.80 \mathrm{E}+03$ & $-1.435178000000 \mathrm{E}+03$ & $-1.435178000000 \mathrm{E}+03$ & \\
\hline 41 & agg2 & $1.58 \mathrm{E}+05$ & $6.71 E+03$ & $-2.023925235598 \mathrm{E}+07$ & $-2.023925235598 \mathrm{E}+07$ & \\
\hline 42 & agg3 & $3.43 E+05$ & $1.40 \mathrm{E}+04$ & $1.031211593509 \mathrm{E}+07$ & $1.031211593509 \mathrm{E}+07$ & \\
\hline 43 & $\operatorname{scsd} 6$ & $9.31 E+02$ & $9.31 \mathrm{E}+02$ & $5.050000007826 \mathrm{E}+01$ & $5.050000007714 \mathrm{E}+01$ & 2.21 \\
\hline 44 & ship04s & $2.29 \mathrm{E}+03$ & $2.65 \mathrm{E}+02$ & $1.798714700445 E+06$ & $1.798714700445 E+06$ & \\
\hline AS & seba & $3.78 \mathrm{E}+06$ & $1.67 \mathrm{E}+05$ & $1.571160000000 \mathrm{E}+04$ & $1.571160000000 \mathrm{E}+04$ & \\
\hline 46 & tuff & $1.00 \mathrm{E}+08$ & $7.63 E+03$ & $2.921477650936 \mathrm{E}-01$ & $2.921477650936 \mathrm{E}-01$ & \\
\hline 47 & forplan & $8.68 \mathrm{E}+07$ & $7.82 \mathrm{E}+04$ & $-6.642189612722 E+02$ & $-6.642189612722 E+02$ & \\
\hline 48 & bnll & $2.12 E+07$ & $1.37 \mathrm{E}+06$ & $1.977629561523 \mathrm{E}+03$ & $1.977629561523 \mathrm{E}+03$ & \\
\hline 49 & pilot4 & $5.48 \mathrm{E}+10$ & $4.95 \mathrm{E}+06$ & $-2.581139259543 E+03$ & $-2.581139258885 E+03$ & $2.549 \mathrm{E}-10$ \\
\hline 50 & scfxum2 & $4.09 \mathrm{E}+06$ & $3.95 \mathrm{E}+03$ & $3.666026156500 \mathrm{E}+04$ & $3.666026156500 \mathrm{E}+04$ & \\
\hline
\end{tabular}




\section{TABLE II}

\section{Stability of Optimal Bases}

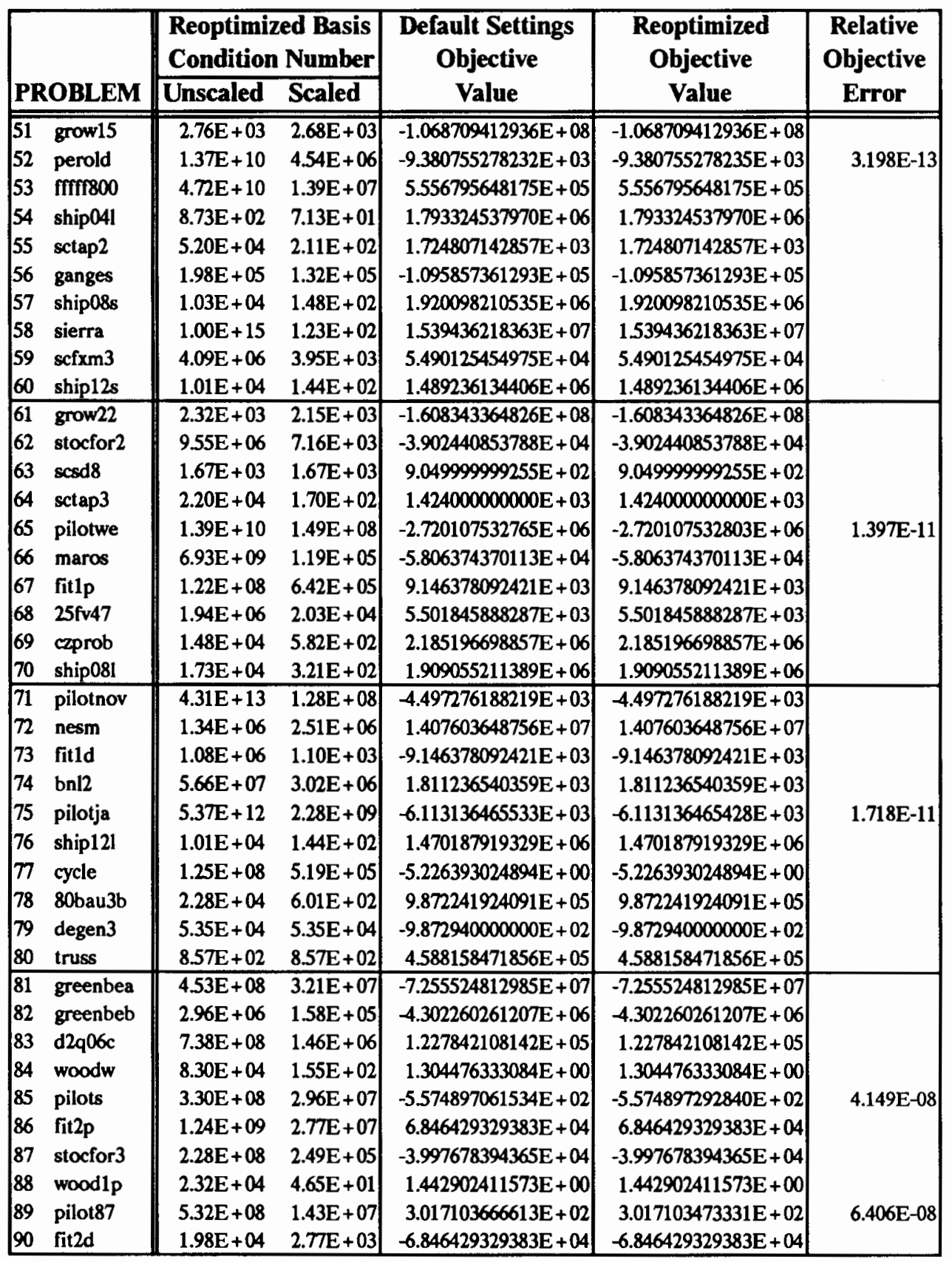


TABLE III

Residuals for Optimal Bases

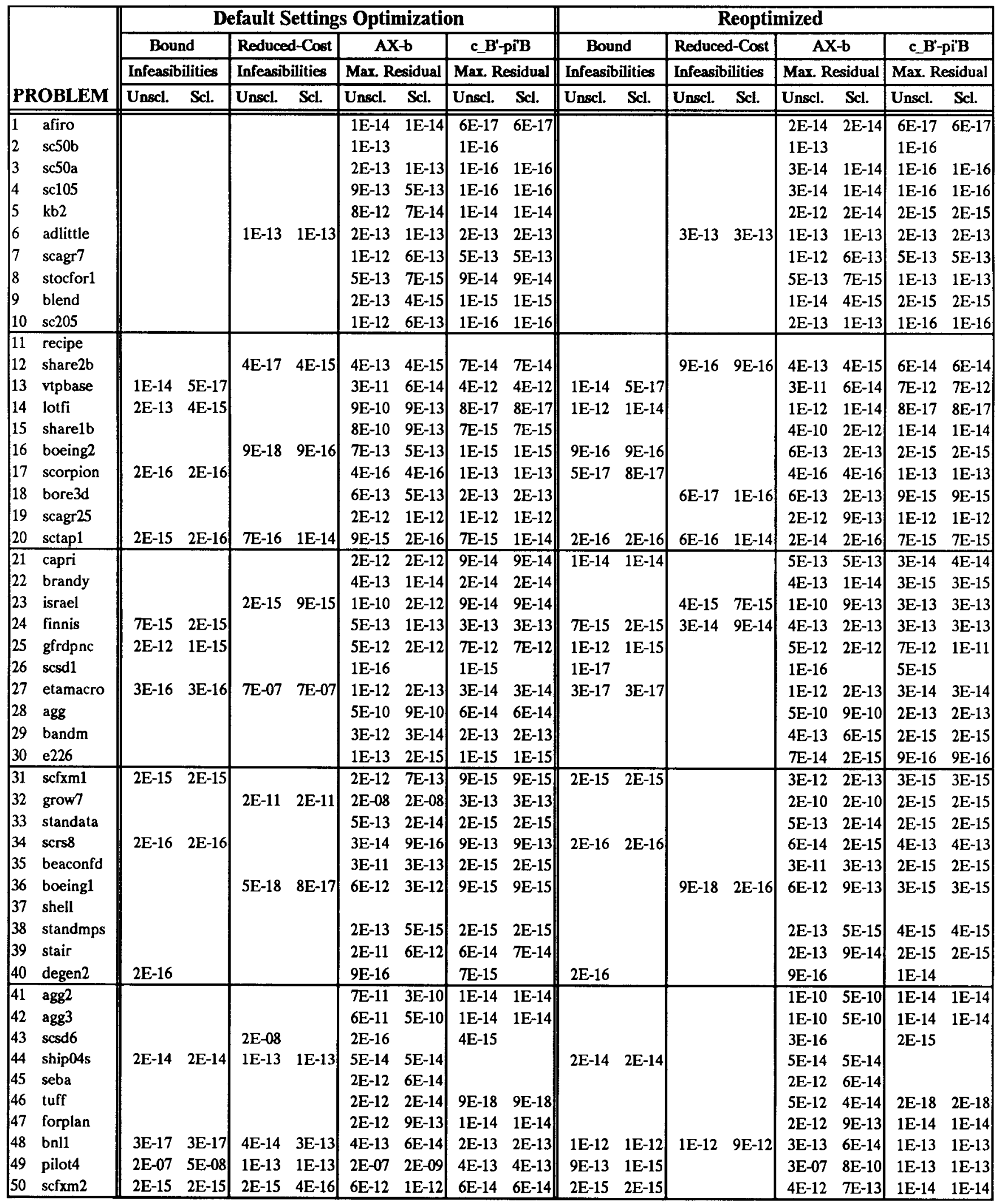




\section{TABLE III}

Residuals for Optimal Bases

\begin{tabular}{|c|c|c|c|c|c|c|c|c|c|c|c|c|c|c|c|c|c|}
\hline & \multicolumn{8}{|c|}{ Default Settings Optimization } & \multicolumn{8}{|c|}{ Reoptimized } \\
\hline & & \multicolumn{2}{|c|}{ Bound } & \multicolumn{2}{|c|}{ Reduced-Cost } & \multicolumn{2}{|c|}{$\mathbf{A X - b}$} & \multicolumn{2}{|c|}{ c_B'-pi'B } & \multicolumn{2}{|c|}{ Bound } & \multicolumn{2}{|c|}{ Reduced-Cost } & \multicolumn{2}{|c|}{$\mathbf{A X}-\mathbf{b}$} & \multicolumn{2}{|c|}{ c_B'-pi'B } \\
\hline & & \multicolumn{2}{|c|}{ Infeasibilities } & \multicolumn{2}{|c|}{ Infeasibilities } & \multicolumn{2}{|c|}{ Max. Residual } & \multicolumn{2}{|c|}{ Mar. Residual } & \multicolumn{2}{|c|}{ Infeasibilities } & \multicolumn{2}{|c|}{ Infeasibilities } & \multicolumn{2}{|c|}{ Max. Residual } & \multicolumn{2}{|c|}{ Max. Residual } \\
\hline & & Unscl. & Scl. & Unscl. & Scl. & Unscl. & Scl. & Unscl. & Scl. & Unscl. & Scl. & Unscl. & Scl. & Unscl. & Sel. & Unscl. & Scl. \\
\hline 51 & ow15 & & & $\overline{7 \mathrm{E}-12}$ & $7 \mathrm{E}-12$ & $3 \mathrm{E}-09$ & $3 \mathrm{E}-09$ & $6 \mathrm{E}-12$ & $7 \mathrm{E}-12$ & & & $2 \mathrm{E}-13$ & $2 \mathrm{E}-13$ & $2 \mathrm{E}-09$ & $2 \mathrm{E}-09$ & $2 \mathrm{E}-13$ & $2 \mathrm{E}-13$ \\
\hline J & perold & $4 \mathrm{E}-12$ & 9E-15 & $1 \mathrm{E}-13$ & $1 \mathrm{E}-13$ & $2 \mathrm{E}-06$ & $2 \mathrm{E}-09$ & $1 E-11$ & $1 E-11$ & $4 \mathrm{E}-12$ & $9 \mathrm{E}-15$ & $8 E-14$ & $1 E-13$ & $3 E-09$ & $3 \mathrm{E}-12$ & $1 \mathrm{E}-13$ & $1 E-13$ \\
\hline & Iffr 800 & & & & & $1 \mathrm{E}-10$ & $2 \mathrm{E}-11$ & $3 E-11$ & $3 \mathrm{E}-11$ & & & & & $2 \mathrm{E}-10$ & $3 \mathrm{E}-11$ & $3 E-11$ & $3 E-11$ \\
\hline & hip041 & & & $4 E-13$ & $4 E-13$ & $2 E-14$ & $3 E-14$ & & & & & $4 \mathrm{E}-13$ & $4 \mathrm{E}-13$ & $2 \mathrm{E}-14$ & $3 E-14$ & & \\
\hline o. & ctap2 & & & $5 E-16$ & $1 \mathrm{E}-14$ & $9 \mathrm{E}-15$ & $2 \mathrm{E}-16$ & $7 \mathrm{E}-15$ & $1 \mathrm{E}-14$ & $6 \mathrm{E}-16$ & $6 \mathrm{E}-16$ & $4 \mathrm{E}-16$ & $7 \mathrm{E}-15$ & $9 E-15$ & $2 E-16$ & $1 E-14$ & $1 E-14$ \\
\hline 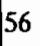 & ganges & & & & & $1 \mathrm{E}-11$ & $1 E-11$ & $6 \mathrm{E}-14$ & $6 \mathrm{E}-14$ & & & $1 \mathrm{E}-13$ & $1 \mathrm{E}-13$ & $1 \mathrm{E}-11$ & $1 \mathrm{E}-11$ & $8 \mathrm{E}-14$ & $8 E-14$ \\
\hline 57 & ship08s & $5 E-16$ & $5 E-16$ & & & $2 \mathrm{E}-14$ & $2 \mathrm{E}-14$ & & & $5 E-16$ & $5 \mathrm{E}-16$ & & & $2 \mathrm{E}-14$ & $2 \mathrm{E}-14$ & & \\
\hline D० & erra & $2 E-13$ & $2 \mathrm{E}-13$ & $6 \mathrm{E}-07$ & $6 \mathrm{E}-07$ & $9 \mathrm{E}-13$ & $9 \mathrm{E}-13$ & $4 \mathrm{E}-12$ & $4 E-12$ & $2 \mathrm{E}-13$ & $2 \mathrm{E}-13$ & $4 E-12$ & $4 E-12$ & $9 \mathrm{E}-13$ & $9 E-13$ & $4 \mathrm{E}-12$ & $4 E-12$ \\
\hline 59 & $\operatorname{scfxm} 3$ & $2 E-15$ & $2 \mathrm{E}-15$ & $2 \mathrm{E}-15$ & $7 \mathrm{E}-16$ & $4 E-12$ & $7 \mathrm{E}-13$ & $1 \mathrm{E}-13$ & $1 \mathrm{E}-13$ & $2 \mathrm{E}-15$ & $2 \mathrm{E}-15$ & $2 \mathrm{E}-15$ & $7 \mathrm{E}-16$ & $7 \mathrm{E}-12$ & $7 \mathrm{E}-13$ & $1 \mathrm{E}-14$ & $1 E-14$ \\
\hline 60 & ship12s & $2 \mathrm{E}-15$ & $3 E-14$ & & & 3E-14 & $6 \mathrm{E}-14$ & $9 \mathrm{E}-13$ & $9 \mathrm{E}-13$ & $2 \mathrm{E}-15$ & $4 \mathrm{E}-14$ & & & $3 \mathrm{E}-14$ & $6 \mathrm{E}-14$ & $9 \mathrm{E}-13$ & $9 E-13$ \\
\hline 61 & grow 22 & & & $5 \mathrm{E}-11$ & $5 E-11$ & $3 \mathrm{E}-07$ & $3 \mathrm{E}-07$ & $8 E-12$ & 9E-12 & & & $7 \mathrm{E}-12$ & $7 \mathrm{E}-12$ & $2 \mathrm{E}-08$ & $2 \mathrm{E}-08$ & $5 E-12$ & $7 \mathrm{E}-12$ \\
\hline 62 & stocfor 2 & $7 \mathrm{E}-16$ & $7 \mathrm{E}-16$ & & & $5 E-12$ & $2 \mathrm{E}-14$ & $6 \mathrm{E}-14$ & $6 \mathrm{E}-14$ & $7 \mathrm{E}-16$ & $7 \mathrm{E}-16$ & & & $2 \mathrm{E}-12$ & $1 \mathrm{E}-14$ & $9 E-14$ & 9E-14 \\
\hline 63 & $\operatorname{scsd} 8$ & $4 \mathrm{E}-16$ & & & & $4 \mathrm{E}-15$ & & $1 \mathrm{E}-14$ & & $2 \mathrm{E}-16$ & & & & $2 \mathrm{E}-15$ & & $2 E-14$ & \\
\hline 64 & sctap3 & & & $4 E-16$ & 7E-15 & $9 E-15$ & 2E-16 & $2 \mathrm{E}-14$ & $2 \mathrm{E}-14$ & & & $4 E-16$ & $7 \mathrm{E}-15$ & $9 E-15$ & $2 E-16$ & $1 E-14$ & $1 E-14$ \\
\hline os & pilotwe & $7 \mathrm{E}-12$ & $2 \mathrm{E}-15$ & & & 4E-07 & 5E-09 & $6 \mathrm{E}-10$ & $6 \mathrm{E}-10$ & $7 \mathrm{E}-12$ & $2 \mathrm{E}-15$ & & & $4 E-05$ & 8E-09 & $1 E-09$ & $1 E-09$ \\
\hline 66 & maros & $2 \mathrm{E}-10$ & $1 \mathrm{E}-14$ & $5 E-13$ & $5 E-13$ & $5 E-10$ & $5 E-12$ & $3 E-12$ & $3 E-12$ & $8 \mathrm{E}-13$ & $1 \mathrm{E}-13$ & & & $9 \mathrm{E}-10$ & $9 \mathrm{E}-13$ & $2 E-14$ & $3 E-14$ \\
\hline 67 & fitlp & & & & & $5 E-14$ & $2 E-16$ & $2 E-12$ & $2 \mathrm{E}-12$ & & & & & $5 \mathrm{E}-14$ & $2 \mathrm{E}-16$ & $2 \mathrm{E}-12$ & 2E-12 \\
\hline 68 & $25 f v 47$ & & & & & $1 \mathrm{E}-11$ & $1 \mathrm{E}-11$ & $2 E-13$ & $2 \mathrm{E}-13$ & & & & & $2 \mathrm{E}-12$ & $3 E-13$ & $2 E-14$ & $2 E-14$ \\
\hline 6 & & & & & & $6 \mathrm{E}-13$ & $6 \mathrm{E}-13$ & $3 E-14$ & $3 E-14$ & & & & & $2 \mathrm{E}-12$ & $2 \mathrm{E}-12$ & $3 E-14$ & $3 E-14$ \\
\hline 70 & ship081 & $4 \mathrm{E}-15$ & $4 \mathrm{E}-15$ & & & $4 \mathrm{E}-14$ & $4 \mathrm{E}-14$ & $9 \mathrm{E}-13$ & $9 \mathrm{E}-13$ & $4 \mathrm{E}-15$ & $4 \mathrm{E}-15$ & & & 2E-14 & $2 \mathrm{E}-14$ & $9 \mathrm{E}-13$ & 9E-13 \\
\hline 71 & pilotnd & -16 & 1E-16 & & & $6 \mathrm{E}-09$ & 1E-10 & $3 \mathrm{E}-16$ & $3 \mathrm{E}-16$ & E-12 & $2 \mathrm{E}-15$ & & & 5E-07 & $4 E-09$ & $1 \mathrm{E}-16$ & $1 E-16$ \\
\hline 72 & nesm & $1 \mathrm{E}-08$ & $1 E-08$ & $2 \mathrm{E}-08$ & 2E-07 & $1 \mathrm{E}-11$ & $7 \mathrm{E}-13$ & 2E-12 & $2 \mathrm{E}-12$ & $9 E-13$ & $8 \mathrm{E}-14$ & $7 \mathrm{E}-11$ & $7 \mathrm{E}-11$ & $3 E-12$ & $9 E-13$ & $8 E-13$ & $8 E-13$ \\
\hline 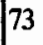 & fitld & & & & & $7 E-13$ & $9 \mathrm{E}-16$ & $7 \mathrm{E}-15$ & $7 \mathrm{E}-15$ & & & & & $7 E-13$ & $9 E-16$ & $7 \mathrm{E}-15$ & $7 \mathrm{E}-15$ \\
\hline 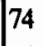 & bn12 & $4 \mathrm{E}-14$ & $4 \mathrm{E}-14$ & $7 \mathrm{E}-07$ & $7 \mathrm{E}-07$ & $2 E-13$ & $8 E-14$ & $1 \mathrm{E}-14$ & $1 \mathrm{E}-14$ & $2 E-14$ & $2 \mathrm{E}-14$ & $1 E-15$ & $4 \mathrm{E}-15$ & $2 E-13$ & $1 \mathrm{E}-13$ & $1 E-14$ & 1E-14 \\
\hline - & pilotj & $7 \mathrm{E}-12$ & $3 E-16$ & $3 \mathrm{E}-15$ & $3 \mathrm{E}-15$ & $7 \mathrm{E}-05$ & $3 E-09$ & 2E-12 & 2E-12 & $7 \mathrm{E}-12$ & $3 E-16$ & $2 E-13$ & $2 E-13$ & 2E-04 & $6 \mathrm{E}-09$ & $2 \mathrm{E}-11$ & 2E-11 \\
\hline 16 & ship121 & $3 E-15$ & $3 E-15$ & & & $5 \mathrm{E}-14$ & $6 \mathrm{E}-14$ & $5 E-13$ & $5 E-13$ & $3 \mathrm{E}-15$ & $3 E-15$ & & & $5 E-14$ & $6 E-14$ & & \\
\hline 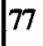 & cycle & & & & & $3 E-12$ & $3 E-14$ & $3 E-17$ & $3 E-17$ & $3 E-13$ & $7 \mathrm{E}-15$ & $4 E-19$ & $4 E-19$ & $2 \mathrm{E}-12$ & $6 \mathrm{E}-14$ & $3 E-17$ & $3 \mathrm{E}-17$ \\
\hline 78 & 80bau3b & $2 E-13$ & 1E-13 & & & $2 E-12$ & $2 E-12$ & $3 E-14$ & $3 E-14$ & $2 E-13$ & $1 E-13$ & & & 2E-12 & $2 \mathrm{E}-12$ & $1 E-14$ & $1 \mathrm{E}-14$ \\
\hline 79 & degen 3 & $4 \mathrm{E}-15$ & & & & $1 E-14$ & & $4 E-15$ & & $2 E-15$ & & $4 E-16$ & & $7 \mathrm{E}-15$ & & $4 E-15$ & \\
\hline 80 & truss & $4 \mathrm{E}-15$ & & & & $2 \mathrm{E}-13$ & & $2 \mathrm{E}-12$ & & 4E-15 & & & & $2 \mathrm{E}-13$ & & 2E-12 & \\
\hline 81 & greenbea & $2 \mathrm{E}-13$ & $2 \mathrm{E}-13$ & $1 \mathrm{E}-12$ & $1 \mathrm{E}-12$ & $1 \mathrm{E}-08$ & $1 E-08$ & $2 \mathrm{E}-11$ & 2E-11 & $2 \mathrm{E}-13$ & $2 \mathrm{E}-13$ & $1 E-12$ & $1 \mathrm{E}-12$ & $1 \mathrm{E}-08$ & $1 \mathrm{E}-08$ & $3 E-11$ & $3 \mathrm{E}-11$ \\
\hline 82 & greenbe & $1 E-13$ & $1 \mathrm{E}-13$ & $7 \mathrm{E}-17$ & $1 \mathrm{E}-16$ & $7 \mathrm{E}-12$ & $7 \mathrm{E}-12$ & $4 \mathrm{E}-12$ & $4 E-12$ & $1 \mathrm{E}-13$ & 1E-13 & $3 E-13$ & $3 E-13$ & $3 E-12$ & $3 \mathrm{E}-12$ & $4 \mathrm{E}-12$ & $4 \mathrm{E}-12$ \\
\hline 83 & $\mathrm{~d} 2 \mathrm{q} 06 \mathrm{c}$ & & & $2 \mathrm{E}-15$ & $2 \mathrm{E}-15$ & $8 E-10$ & $7 \mathrm{E}-12$ & 2E-13 & 2E-13 & & & $6 \mathrm{E}-16$ & $2 E-15$ & $5 E-10$ & $9 E-13$ & $2 E-14$ & $2 \mathrm{E}-14$ \\
\hline 84 & woodw & & & & & $1 E-13$ & $1 E-16$ & $3 E-16$ & $3 E-16$ & & & & & $1 E-13$ & $1 \mathrm{E}-16$ & $3 E-16$ & $3 E-16$ \\
\hline 85 & pilots & $3 E-08$ & $2 \mathrm{E}-08$ & $1 \mathrm{E}-08$ & $5 E-08$ & $6 \mathrm{E}-10$ & $8 E-11$ & $2 \mathrm{E}-12$ & $2 \mathrm{E}-12$ & $1 E-13$ & $2 E-14$ & $2 \mathrm{E}-16$ & $2 E-16$ & $7 E-10$ & $7 \mathrm{E}-11$ & $9 E-14$ & $9 E-14$ \\
\hline 86 & fit $2 p$ & $1 E-14$ & $6 \mathrm{E}-16$ & & & $7 E-14$ & $4 E-15$ & $3 E-11$ & $3 E-11$ & $1 E-14$ & $6 \mathrm{E}-16$ & & & $7 \mathrm{E}-14$ & $4 \mathrm{E}-15$ & $3 \mathrm{E}-11$ & $3 E-11$ \\
\hline 87 & stocror3 & $1 \mathrm{E}-15$ & $1 \mathrm{E}-15$ & & & $2 \mathrm{E}-11$ & TE-14 & $4 \mathrm{E}-14$ & $4 E-14$ & $3 \mathrm{E}-15$ & $3 E-15$ & & & $9 \mathrm{E}-12$ & $3 E-14$ & $1 \mathrm{E}-13$ & $1 \mathrm{E}-13$ \\
\hline 88 & woodlp & $4 E-14$ & $4 \mathrm{E}-17$ & & & $1 E-13$ & $3 E-16$ & $4 \mathrm{E}-15$ & 4E-15 & $2 E-14$ & $2 \mathrm{E}-17$ & & & $9 E-14$ & $7 \mathrm{E}-16$ & $3 E-15$ & $3 E-15$ \\
\hline 89 & pilot87 & $2 E-12$ & $1 E-13$ & 2E-07 & $4 E-07$ & $6 E-08$ & $6 \mathrm{E}-09$ & $2 E-12$ & $2 E-12$ & $2 E-12$ & $1 E-13$ & & & $1 E-09$ & $1 \mathrm{E}-10$ & $9 E-15$ & $1 E-14$ \\
\hline 90 & fit $2 d$ & & & & & $1 \mathrm{E}-13$ & $4 \mathrm{E}-16$ & 1E-14 & $1 \mathrm{E}-1$ & & & & & $1 \mathrm{E}-13$ & 4E-16 & $1 \mathrm{E}-14$ & 1E-14 \\
\hline
\end{tabular}




\section{Part II: The Initial Basis}

\section{Constructing an Initial Basis}

The simplex method requires as input a feasible basis. If no such basis is available, it is standard to begin by constructing some sort of auxiliary or "phase I" problem. This auxiliary problem is then solved, and the resulting basis (in the case of feasibility) is used as the starting basis to solve the original problem.

In this section four alternate initial bases are described. The first is the classical all "artificial" basis, essentially that presented in most texts on linear programming. The next two bases, the feasible slack and slack, can be viewed as the natural next steps in an attempt to eliminate the use of artificial variables. Indeed, they were the intermediate steps that led to the development of the initial basis that is currently used in CPLEX. That basis is described last.

Suppose that $\left(B, N_{l}, N_{u}\right)$ satisfies all the conditions for a basis, except feasibility. For $j=1, \ldots, n$, define

$$
p_{j}(x)=\left\{\begin{array}{ccc}
x-u_{j} & \text { if } & x>u_{j}, \\
0 & \text { if } & l_{j} \leq x \leq u_{j},
\end{array}\right. \text { and }
$$

The associated phase $I$ problem is piecewise linear: ${ }^{5}$

$$
\begin{array}{ll}
\operatorname{minimize} & \sum_{j=1}^{n} p_{j}\left(x_{j}\right) \\
\text { s.t. } & A x=b
\end{array}
$$

The basis $B$ is obviously feasible for (4), and (1) has a feasible solution if and only if the optimal objective value of (4) is 0 . It should be noted that the initial values of the basic variables in the slack and CPLEX bases, described below, may violate their bounds, and so dictate the need for viewing the phase I problem as piecewise linear. On the other hand, the artificial and feasible slack bases result in initial optimization problems that are ordinary LP's.

\section{All Artificial Basis}

The simplest approach to finding an initial basis is to begin with an all "artificial" basis. Let

$$
\begin{aligned}
& N=\{1, \ldots, n\}, \\
& N_{l}=\left\{j \in N \backslash\left(N_{f r} \cup N_{f x}\right):\left|l_{j}\right| \leq\left|u_{j}\right|\right\}, \\
& N_{u}=\left\{j \in N \backslash\left(N_{f r} \cup N_{f x}\right):\left|l_{j}\right|>\left|u_{j}\right|\right\}, \text { and } \\
& \tilde{b}=b-A_{N} X_{N},
\end{aligned}
$$
it.

${ }^{5}$ While (4) is not an LP, it is easy to see that all of the definitions in section 2 can be applied to 
and consider the problem

$$
\begin{array}{ll}
\operatorname{minimize} & e^{T} z \\
\text { s.t. } & A x+D z=b \\
& l \leq x \leq u \\
& z \geq 0
\end{array}
$$

where $z^{T}=\left(x_{n+1}, \ldots, x_{n+m}\right), e^{T}=(1, \ldots, 1)$ and $D=\operatorname{diag}\left(\delta_{1}, \ldots, \delta_{m}\right)$ with

$$
\delta_{i}= \begin{cases}+1 & \text { if } \bar{b}_{i} \geq 0, \text { and } \\ -1 & \text { otherwise }\end{cases}
$$

The variables $z$ are called artificial variables. Clearly (1) is feasible if and only if the optimum value of $(6)$ is 0 .

The artificial basis for $(6)$ is $\left(B, N_{l}, N_{u}\right)$ where $B=(n+1, \ldots, n+m)$, and $N_{l}$ and $N_{u}$ are as given in (5). $B$ is clearly feasible for (6).

\section{Feasible Slack Basis}

The typical LP, as specified by the user, contains some inequality constraints:

$$
\begin{array}{ll}
\operatorname{minimize} & c^{T} x \\
\text { s.t. } & A_{1} x \leq b_{1} \\
& A_{2} x \geq b_{2} \\
& A_{3} x=b_{3} \\
& l \leq x \leq u
\end{array}
$$

Assume that $b_{i}$ is an $m_{i}$-vector for $i=1,2,3\left(m=m_{1}+m_{2}+m_{3}\right)$. Problem (7) is converted to form (1) by adding slack variables $s_{1}^{T}=\left(x_{n+1}, \ldots, x_{n+m_{1}}\right)$ and $s_{2}^{T}=$ $\left(x_{n+m_{1}+1}, \ldots, x_{n+m_{1}+m_{2}}\right)$ :

$$
\begin{array}{ll}
\operatorname{minimize} & c^{T} x \\
\text { s.t. } & A_{1} x+s_{1}=b_{1} \\
& A_{2} x-s_{2}=b_{2} \\
& A_{3} x=b_{3} \\
& l \leq x \leq u \\
& s_{1} \geq 0, s_{2} \geq 0
\end{array}
$$

For this problem, the first step in constructing a feasible slack basis is to construct the artificial basis, noting that all slack variables will initially be in $N_{l}$. Then, for $i \in\left\{1, \ldots, m_{1}\right\}$ if $\delta_{i}=1$, and for $i \in\left\{m_{1}+1, \ldots, m_{1}+m_{2}\right\}$ if $\delta_{i}=-1$, the artificial $x_{n+m_{1}+m_{2}+i}$ variable is replaced in $B$ by the slack $x_{m+i}$. In other words, available slacks are used so long as they are initially nonnegative. 


\section{Slack Basis}

In this approach, all available slacks are used, independent of feasibility. Consider the problem

$$
\begin{array}{ll}
\operatorname{minimize} & \sum_{j=1}^{n+m} p_{j}\left(x_{j}\right) \\
\text { s.t. } & A_{1} x+s_{1}=b_{1} \\
& A_{2} x-s_{2}=b_{2} \\
& A_{3} x+z=b_{3}
\end{array}
$$

where the $p_{j}$ are given by (3), using the bounds $s_{1} \geq 0, s_{2} \geq 0$ and $z=0$ for the slacks and artificials, respectively. The initial basis is $\left(B, N_{l}, N_{u}\right)$ where $B=$ $(n+1, \ldots, n+m)$, and $N_{l}$ and $N_{u}$ are as given in (5).

\section{CPLEX Basis}

The procedure described below is more complicated than the three described above. However, its implementation is quite simple. The essential idea is to construct a sparse, well-behaved basis, with as much freedom as possible, and as few artificials as possible. First, a preference order for the variables is constructed; this order is then used to construct the basis. As the computational results will show, using the initial basis constructed in this way can greatly reduce the number of iterations, especially for "easy problems." For harder problems, it is generally less effective.

It is perhaps natural to think that just the opposite would be true, that the starting basis should be more effective for harder problems. Indeed, precisely that consideration is the main motivation for developing so-called "crash" procedures, some of which can be remarkably effective in dealing with particular problem structures, such as those arising in multiperiod models. However, in the opinion of this author, the CPLEX initial basis is best viewed not as a crash, but simply as a default starting basis. In general, trying to guess a correct basis for a problem with unknown origins can have disastrous consequences. The goal of the CPLEX basis is not so much to try to find variables that are likely to be in any optimal basis, but to avoid the work of removing artificial variables, and avoid the effect that their restrictive bounds have on the optimization.

Assume that the given problem has the form (7) and has been converted to form (1) by adding slack variables. The preference order for variables is determined as follows. Define the following sets, where $C_{i}$ will be "preferred" to $C_{i+1}(i=1,2,3)$ :

$$
\begin{aligned}
& C_{1}=\left\{n+1, \ldots, n+m_{1}+m_{2}\right\}, \\
& C_{2}=\left\{j: x_{j} \text { free }\right\} \\
& C_{3}=\left\{j \leq n: \text { exactly one of } l_{j} \text { and } u_{j} \text { is finite }\right\}, \text { and } \\
& C_{4}=\left\{j:-\infty<l_{j}, u_{j}<+\infty\right\} .
\end{aligned}
$$

Every variable falls into one of the above sets. Note that $C_{1}$ is just the set of indices of the slack variables. Slack variables are preferred to free variables because of the sparsity and numerical properties of the corresponding matrix columns. 
For $j \in\left\{1, \ldots, n+m_{1}+m_{2}\right\}$, define a penalty $\bar{q}_{j}$ by

$$
\bar{q}_{j}=\left\{\begin{array}{cl}
0 & \text { if } j \in C_{2}, \\
l_{j} & \text { if } j \in C_{3} \text { and } u_{j}=+\infty, \\
-u_{j} & \text { if } j \in C_{3} \text { and } l_{j}=-\infty, \text { and } \\
l_{j}-u_{j} & \text { if } j \in C_{4} .
\end{array}\right.
$$

Let $\gamma=\max \left\{\left|c_{j}\right|: 1 \leq j \leq n\right\}$ and define

$$
c_{\max }=\left\{\begin{array}{cl}
1000 \gamma & \text { if } \gamma \neq 0 \\
1 & \text { otherwise }
\end{array}\right.
$$

Finally, for $j \in\{1, \ldots, n\}$, define

$$
q_{j}=\bar{q}_{j}+c_{j} / c_{\max }
$$

The variables within $C_{2}, C_{3}$ and $C_{4}$ are sorted in ascending order of $q_{j}$ value and the lists are concatenated into a single ordered set $C=\left(j_{1}, \ldots, j_{n}\right)$. The sorting has the effect of placing the variables with the most "freedom" at the start, or preferred-end, of the list, using the objective function to break ties.

The basis $B$ can now be constructed. After the construction is complete, the indices in $N \backslash\left(B \cup N_{f r} \cup N_{f x}\right)$ are assigned to $N_{l}$ and $N_{u}$ according to (5). As previously noted, the constraint matrix is always scaled by CPLEX. The important feature of the scaling is that, after scaling, the maximum absolute value in every non-zero row and column is 1 .

Step 1: For $i=1, \ldots, m_{1}+m_{2}$, set

$$
\begin{aligned}
& I_{i} \leftarrow 1, \text { and } \\
& r_{i} \leftarrow 1 .
\end{aligned}
$$

Set $B \leftarrow\left\{n+1, \ldots, n+m_{1}+m_{2}\right\}$. For $i=m_{1}+m_{2}+1, \ldots, m$, set $I_{i} \leftarrow 0$ and $r_{i} \leftarrow 0$. For $i=1, \ldots, m$ set $v_{i} \leftarrow+\infty$.

Step 2: Let $C=\left(j_{1}, \ldots, j_{n}\right)$. For $k=1, \ldots, n$, apply the following procedure:

(a) Let $\alpha=\max \left\{\left|A_{l j_{k}}\right|: r_{l}=0\right\}$. If $\alpha \geq 0.99$, let $l^{\prime}$ be such that $\alpha=\left|A_{l^{\prime} j_{k}}\right|$ and $r_{l^{\prime}}=0$. Set

$$
\begin{aligned}
& B \leftarrow B \cup\left\{j_{k}\right\} \\
& I_{l^{\prime}} \leftarrow 1, \\
& v_{l^{\prime}} \leftarrow \alpha, \text { and } \\
& r_{l} \leftarrow r_{l}+1 \text { for all } l \text { such that }\left|A_{l j_{k}}\right| \neq 0
\end{aligned}
$$

Continue to the next $k$. 
(b) If $\left|A_{l j_{k}}\right|>0.01 v_{l}$ for some $l$, continue to the next $k$; otherwise, let $\alpha=$ $\max \left\{\left|A_{l j_{k}}\right|: I_{l}=0\right\}$. If $\alpha=0$, continue to the next $k$; otherwise, let $l^{\prime}$ be such that $I_{l^{\prime}}=0$ and $\alpha=\left|A_{l^{\prime} j_{k}}\right|$. Set

$$
\begin{aligned}
& B \leftarrow B \cup\left\{j_{k}\right\}, \\
& I_{l^{\prime}} \leftarrow 1, \\
& v_{l^{\prime}} \leftarrow \alpha, \text { and } \\
& r_{l} \leftarrow r_{l}+1 \text { for all } l \text { such that }\left|A_{l j_{k}}\right| \neq 0 .
\end{aligned}
$$

Step 3: For $i=m_{1}+m_{2}+1, \ldots, m$, if $I_{i}=0$, set $B \leftarrow B \cup\left\{n+m_{1}+m_{2}+i\right\}$ 一that is, add an artificial variable to cover each remaining uncovered row.

The $v$ values in the above construction can be thought of as pseudo pivot values, and the condition that $\left|A_{l j_{i}}\right| \leq 0.01 v_{l}$ in Step 2(b) as enforcing an approximate lower triangularity. Indeed, if 0.01 is replaced by 0 , and $v$ is initialized to any finite number for the slacks (Step 1), then the resulting matrix $\boldsymbol{B}$ is lower triangular. In a similar vein, if $v$ is initialized to 1 for the slacks, and 0.99 is replaced by 1 in Step 2(a), then, it is easy to prove that all $v$ values are 1 .

\section{Computational Results}

All tests were run on a $40 \mathrm{Mhz}$ SPARCstation 2. CPLEX is written entirely in C and was compiled using the flags '-O4 -cg89 -libmil -dalign' and the bundled Sun C compiler.

The CPLEX procedure for constructing an initial basis may be summarized as follows. It begins by placing all available slack variables in the basis. The columns of the constraint matrix are then ordered based on the "distance" between the bounds. Using this ordering, a heuristic factorization routine is applied (Step 2 at the end of the last section) to find "pivot" elements covering equality rows, those rows not covered by the slacks.

The inclusion of all slacks in the initial basis is motivated by the sparsity and "numerical stability" of these columns together with the expectation that a significant fraction will be in the optimal basis. The results in Table IV show that, for the netlib problems an average of $52.9 \%$ of the slacks were used in the optimal bases. For only six of the problems, including pilots and pilot87, was the percentage under 10.

The heuristic factorization was very successful in eliminating artificial variables. An average of only $13.1 \%$ of equality rows were covered by artificials in the initial bases. Almost none of the artificials remained in the optimal bases. One exception was 'cycle', where 130 artificial variables remained in the optimal basis, presumably due to redundancy among the equality constraints.

Each of the 90 netlib problems was solved using each of the four types of initial bases introduced in section 4: artificial (A), feasible slack (FS), slack (S) and CPLEX 
(C). All runs were made in batch mode on a standalone system with no other userjobs running. Default settings were used throughout in CPLEX. Only the initial basis was varied.

The timings reported are the user times returned by the 'times' library function. Totals for phase I iterations, total iterations and run times are reported at the bottom of the table. These numbers are dominated by several of the more difficult problems, and so are not as useful as one might hope. However, they do clearly indicate that the artificial basis is inferior to the CPLEX basis. As one might expect, the biggest part of this advantage comes in phase I. In addition, the improvement in total iterations is significantly greater than the improvement in run time-the CPLEX initial basis, though relatively sparse, is certainly more expensive to handle than an identity basis.

In an attempt to equalize the effect of the difficult problems, two kinds of ratios were added to Table V: ratios of the total iterations required by each of the three simpler bases divided by the iterations required by the CPLEX basis, and corresponding ratios for run times. Ratios for phase I iterations would not have been meaningful. Averages are presented at the bottom of the table. These numbers can be misleading if a sizeable number of the ratios are significantly less than 1.0. That, however, is not the case here. The CPLEX basis leads to slower solution times in 20 of 90 cases. Of those, 12 are by $10 \%$ or less and only 1 by more than $30 \%$. The average improvement of CPLEX over the $\mathrm{S}$ basis is about $35 \%$.

For problems that are difficult, the CPLEX basis is typically not a significant win. Ignoring bounds, a basis has size equal to the number of rows. Thus, a number of iterations approximating the number of rows can be interpreted as good performance: With no reasonable expectation that we can guess optimal columns in advance, it will take that many iterations just to pivot in the columns of an optimal basis.

Of the larger problems, stocfor3 is easy in the above sense. Even though the linear algebra is not cheap, the number of iterations is less than the number of rows, and the CPLEX basis is a significant improvement. On the other hand, greenbea, greenbeb, $\mathrm{d} 2 \mathrm{q} 06 \mathrm{c}$, pilots, fit $2 \mathrm{p}$ and pilot 87 are not easy. The problem fit $2 \mathrm{~d}$ is not a good candidate for this discussion since a large number of variables must be moved to bounds, and the procedure used to select initial values for nonbasic variables was the same for each of the basis selection procedures. 
TABLE IV

Artificials and Slacks in Optimal Bases

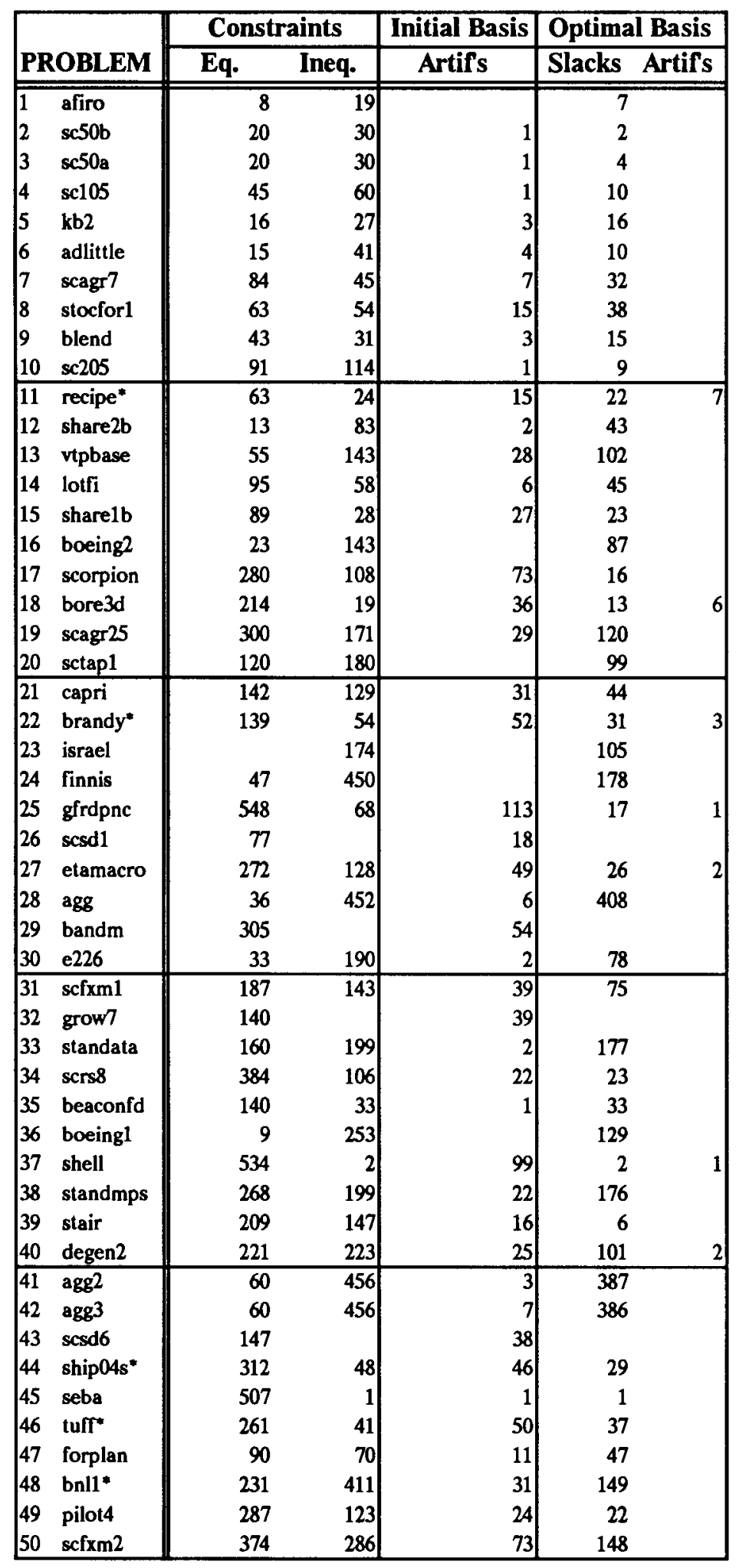




\section{TABLE IV}

\section{Artificials and Slacks in Optimal Bases}

\begin{tabular}{|c|c|c|c|c|c|c|}
\hline \multirow{2}{*}{\multicolumn{2}{|c|}{ PROBLEM }} & \multicolumn{2}{|c|}{ Constraints } & \multirow{2}{*}{\begin{tabular}{|l|} 
Initial Basis \\
Artifs \\
\end{tabular}} & \multicolumn{2}{|c|}{ Optimal Basis } \\
\hline & & Eq. & Ineq. & & \multicolumn{2}{|c|}{ Slacks Artif's } \\
\hline 51 & grow15 & 300 & & 81 & & \\
\hline 52 & perold & 495 & 130 & 82 & 25 & \\
\hline 53 & fffrr800* & 350 & 174 & 17 & 99 & 2 \\
\hline 54 & ship041* & 312 & 48 & 46 & 32 & \\
\hline 55 & sctap2 & 470 & 620 & & 379 & \\
\hline 56 & ganges & 1284 & 25 & 254 & 24 & \\
\hline 57 & ship08s* & 632 & 80 & 74 & 47 & \\
\hline 58 & sierra* & 523 & 699 & 123 & 634 & 10 \\
\hline 59 & scfxm3 & 561 & 429 & 109 & 218 & \\
\hline 60 & ship12s* & 936 & 106 & 133 & 50 & \\
\hline 61 & grow22 & 440 & & 130 & & \\
\hline 62 & stocfor 2 & 1143 & 1014 & 147 & 644 & \\
\hline 63 & sesd8 & 397 & & 72 & & \\
\hline 64 & sctap3 & 620 & 860 & & 516 & \\
\hline 65 & pilotwe & 583 & 139 & 48 & 24 & \\
\hline 66 & maros* & 322 & 522 & 20 & 252 & \\
\hline 67 & fit1p & 627 & & & & \\
\hline 68 & $25 f v 47^{*}$ & 516 & 305 & 117 & 172 & 1 \\
\hline 69 & czprob & 890 & 39 & 10 & 22 & \\
\hline 70 & ship081* & 632 & 80 & 74 & 53 & 1 \\
\hline 71 & pilotnov* & 677 & 274 & 182 & 163 & 5 \\
\hline 72 & nesm & 480 & 94 & 8 & 58 & \\
\hline 73 & fitld & 1 & 23 & & 12 & \\
\hline 74 & bn12 & 1327 & 997 & 212 & 543 & \\
\hline 75 & pilotja & 645 & 279 & 154 & 149 & \\
\hline 76 & ship121* & 936 & 106 & 121 & 46 & \\
\hline 77 & cycle* & 1376 & 514 & 279 & 416 & 131 \\
\hline 78 & 80bau3b & & 2262 & & 133 & \\
\hline 79 & degen 3 & 717 & 786 & 33 & 321 & 2 \\
\hline 80 & truss & 1000 & & 148 & & \\
\hline 81 & greenbea* & 2196 & 193 & 206 & 129 & \\
\hline 82 & greenbeb* & 2196 & 193 & 202 & 122 & 3 \\
\hline 83 & d2q06 $c^{*}$ & 1507 & 664 & 153 & 382 & 2 \\
\hline 84 & woodw & 1085 & 13 & 2 & 9 & 1 \\
\hline 85 & pilots & 233 & 1208 & 11 & 100 & \\
\hline 86 & fit $2 p$ & 3000 & & & & \\
\hline 87 & stocfor3 & 8829 & 7846 & & 4532 & \\
\hline 88 & wood1p* & 243 & 1 & 132 & & 1 \\
\hline 89 & pilot87 & 233 & 1797 & 17 & 150 & \\
\hline 90 & fit2d & 1 & 24 & & 5 & \\
\hline & $\%$ arti & 8 in in & basis & $13.1 \%$ & & \\
\hline & sla & 1 ba & & & $52.9 \%$ & \\
\hline
\end{tabular}




\section{TABLE V}

Comparison of Initial Bases:

Artificial (A), Feasible Slack (FS), Slack (S), CPLEX (C)

\begin{tabular}{|c|c|c|c|c|c|c|c|c|c|c|c|c|c|c|c|c|c|c|c|}
\hline \multirow{2}{*}{\multicolumn{2}{|c|}{ OBLEM }} & \multicolumn{4}{|c|}{ Phase I Iterations } & \multicolumn{4}{|c|}{ Total Iterations } & \multicolumn{4}{|c|}{$\begin{array}{l}\text { Run Times } \\
\text { (seconds) }\end{array}$} & \multicolumn{3}{|c|}{\begin{tabular}{|c|}
$\begin{array}{c}\text { Total Iterations } \\
\text { Ratios }\end{array}$ \\
\end{tabular}} & \multicolumn{3}{|c|}{$\begin{array}{c}\text { Run Time } \\
\text { Ratios }\end{array}$} \\
\hline & & $\mathbf{A}$ & FS & $\mathbf{S}$ & $\bar{C}$ & $\bar{A}$ & FS & $\mathbf{S}$ & $\bar{C}$ & $\mathbf{A}$ & FS & $\mathbf{s}$ & $\mathbf{C}$ & $\mathrm{A} / \mathrm{C}$ & FS/C & $\mathrm{s} / \mathrm{C}$ & $\mathrm{A} / \mathrm{C}$ & FS/C & $\mathrm{s} / \mathrm{C}$ \\
\hline 1 & afiro & 30 & 7 & 7 & 1 & 3 & 18 & 18 & 10 & 0.0 & 0.0 & 0.0 & 0.0 & 8.10 & 1.80 & 1.80 & 1.64 & 1.14 & 1.14 \\
\hline & $\mathrm{sc} 50 \mathrm{~b}$ & 52 & 0 & 0 & 0 & 70 & 48 & 48 & 32 & 0.1 & 0.1 & & 0.1 & 2.19 & 1.50 & 1.50 & 1.33 & 1.18 & 1.18 \\
\hline 3 & sc50a & 43 & 0 & 0 & 0 & 68 & 44 & 44 & 28 & 01 & 0.1 & & 0.0 & 43 & 1.57 & 1.57 & 1.49 & 1.22 & 1.22 \\
\hline 4 & sc105 & 102 & $\mathbf{0}$ & 0 & 0 & 145 & 91 & 91 & 56 & 0.3 & 0.2 & 0.2 & 0.2 & 2.59 & 1.63 & 1.63 & 1.46 & 1.06 & 1.06 \\
\hline 5 & kb2 & 0 & 0 & 0 & 0 & 81 & 59 & 59 & 33 & 0.1 & 0.1 & 0.1 & 0.1 & 2.45 & 1.79 & 1.79 & 1.94 & 1.48 & 1.48 \\
\hline & adlittle & 78 & 29 & 29 & 16 & 116 & 82 & 82 & 94 & 0.2 & 0.1 & & 0.1 & 1.23 & 0.87 & 0.87 & 1.19 & 0.99 & 0.99 \\
\hline & scagr7 & 146 & 117 & 117 & 51 & 179 & 141 & 141 & 87 & 0.3 & 0. & 0.3 & 0.2 & 2.06 & 1.62 & 1.62 & 1.80 & 1.70 & 1.70 \\
\hline 8 & stocfor1 & 163 & 76 & 76 & 15 & 194 & 93 & 93 & 31 & 0.4 & 0.2 & 0.2 & 0.1 & 6.26 & 3.00 & 3.00 & 5.12 & 2.12 & 2.12 \\
\hline 9 & blend & 85 & 0 & 0 & 0 & 100 & 98 & 98 & 58 & 0.2 & 0.2 & 0.2 & 0.2 & 1.72 & 1.69 & 1.69 & 0.92 & 1.03 & 1.03 \\
\hline 10 & sc205 & 190 & 0 & 0 & 0 & 320 & 190 & 190 & 129 & 0.9 & 0.6 & & 0.7 & .48 & 1.47 & 1.47 & 1.43 & 0.96 & 0.96 \\
\hline 11 & recipe & 68 & 51 & 51 & 12 & 94 & 73 & 73 & 41 & 0. & 0.1 & & 0.1 & 2.29 & 1.78 & 1.78 & 1.90 & 1.45 & 1.45 \\
\hline 12 & hare2b & 136 & 69 & 69 & 76 & 181 & 107 & 107 & 111 & 0.4 & 0 & 0.2 & 0.2 & 1.63 & 0.96 & 0.96 & 1.46 & 0.92 & 0.92 \\
\hline 13 & vtpbase & 248 & 100 & 129 & 87 & 274 & 130 & 151 & 114 & 0.7 & 0. & 0. & 0.3 & 2.40 & 1.14 & 1.32 & 2.15 & 0.88 & 1.12 \\
\hline 14 & lotfi & 183 & 105 & 105 & 68 & 284 & 230 & 230 & 199 & 0.6 & 0. & 0. & 0.6 & .43 & 1.16 & 1.16 & 1.07 & 0.75 & 0.75 \\
\hline 5 & ar & 168 & 162 & 162 & 85 & 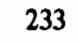 & 240 & 2 & 175 & 0 & & & 0.6 & 33 & 1.37 & 1.37 & 1.11 & 1.13 & 1.13 \\
\hline 16 & boeing & 176 & 122 & 97 & 108 & 242 & 188 & 1 & 178 & 0. & 0 & & 15 & 36 & 1.06 & 0.83 & 1.22 & 0.96 & 0.70 \\
\hline 17 & scorpion & 365 & 317 & 306 & 131 & 399 & 382 & 378 & 168 & 2.0 & 1.7 & 1.5 & 18 & .38 & 2.27 & 2.25 & 2.45 & 2.08 & 1.85 \\
\hline 8 & ore & 213 & $1 s$ & 19 & 91 & 2 & & & 113 & 0 & 0 & & 0.4 & .08 & 1.96 & 1.96 & 1.26 & 1.23 & 1.23 \\
\hline 19 & cagr 25 & 523 & 421 & 418 & 233 & 751 & 565 & 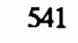 & 400 & 5. & 3. & & 3.3 & 1.88 & 1.41 & 1.35 & 1.61 & 1.13 & 1.06 \\
\hline 20 & sctap1 & 362 & 199 & 180 & 121 & 534 & 350 & 299 & 207 & 2 & 1. & & 0.9 & .58 & 1.69 & 1.44 & 2.39 & 1.55 & 1.22 \\
\hline 21 & capri & 461 & 321 & 321 & 344 & 537 & 414 & 417 & 438 & 2.3 & 1 & 1 & 2.2 & .23 & 0.95 & 0.95 & 1.08 & 0.64 & 0.73 \\
\hline 2 & brandy & 244 & 208 & 208 & 81 & 348 & 326 & 326 & 174 & 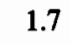 & 1 & 1.5 & 1.1 & .00 & 1.87 & 1.87 & 1.51 & 1.38 & 1.38 \\
\hline 23 & rael & 298 & 8 & 7 & 7 & 464 & 172 & 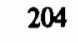 & 204 & 2. & 0 & & .0 & .27 & 0.84 & 1.00 & 2.32 & 0.89 & 0.99 \\
\hline 4 & finnis & 670 & 246 & 255 & 229 & 966 & 461 & 48 & 5 & 5. & 2. & 2 & 3.0 & 1.87 & 0.89 & 0.95 & 1.93 & 0.79 & 0.85 \\
\hline 5 & gfrdpn & 910 & 846 & 846 & 293 & 1156 & 1103 & 1103 & 525 & 6. & 6 & & 3.1 & .20 & 2.10 & 2.10 & 2.00 & 1.97 & 1.97 \\
\hline 26 & $\operatorname{csd} 1$ & 99 & 99 & 99 & 38 & 181 & 181 & 181 & 161 & 0.5 & 0 & & .5 & 12 & 1.12 & 1.12 & 0.90 & 0.90 & 0.90 \\
\hline 7 & etamac & 614 & 511 & 489 & 423 & 93 & 764 & & 718 & 4. & & & 7 & 30 & 06 & 1.01 & 1.28 & 0.99 & 0.93 \\
\hline 8 & agg & 585 & 89 & 77 & 54 & 640 & 136 & 11 & 99 & 3.5 & 0 & 0. & 0.6 & .46 & .37 & 1.17 & 6.76 & 1.24 & 1.10 \\
\hline 29 & bandm & 426 & 426 & 426 & 135 & 594 & 594 & 55 & 283 & 4. & 4. & & 1.9 & 10 & .10 & 2.10 & 2.18 & 18 & 2.18 \\
\hline 30 & e226 & 290 & 01 & 97 & 34 & 449 & & & 3 & & & & 1.9 & & & 1.0 & 1.03 & 1.11 & 0.97 \\
\hline 31 & $\operatorname{sc}\left\{x_{x}\right\}$ & 416 & 258 & 258 & 174 & 520 & 415 & 41 & 32 & 2. & & & 1.5 & 60 & 28 & 1.28 & 1.44 & 1.13 & 1.13 \\
\hline 32 & grow7 & 0 & 0 & 0 & 0 & 309 & 309 & 309 & 275 & 2.2 & 2. & & .5 & 12 & 1.12 & 1.12 & 0.90 & 0.90 & 0.90 \\
\hline 33 & standat & 440 & 17 & 179 & 49 & 522 & 223 & & 153 & 1. & 0 & & 0.7 & 41 & 1.46 & 1.46 & 2.54 & 1.11 & 1.11 \\
\hline 34 & scrs8 & 857 & 8 & 860 & 339 & 1117 & 1148 & 1168 & 561 & 7. & 7. & 7. & 4.6 & 99 & 05 & 2.08 & 1.60 & 1.60 & 1.69 \\
\hline 35 & beac & 16 & 12 & 12 & 0 & 18 & 163 & & 31 & 0. & 0. & & 1 & 10 & 5.26 & 5.26 & 3.58 & 3.25 & 3.25 \\
\hline 36 & boein & 57 & 3 & 34 & 226 & 9 & 710 & & 563 & 4. & 3 & & & 77 & 1.26 & 1.20 & 1.38 & 1.06 & 0.95 \\
\hline 37 & shell & 619 & 6 & 6 & 260 & 1 & 793 & & 494 & & & & & 57 & 1.61 & 1.61 & 1.26 & 1.29 & 1.29 \\
\hline 38 & standn & 585 & 316 & 313 & 209 & 698 & & & 317 & 2 & 1. & & 1.6 & 20 & 44 & 1.40 & 1.70 & 1.10 & 1.10 \\
\hline 39 & stair & 492 & 3 & 3 & 227 & & & & 4 & 9. & 6 & & & & 21 & 1.3 & 1.39 & 0.92 & 1.11 \\
\hline 40 & degen? & 6 & 673 & 659 & 547 & 25 & 1107 & & o>s & 9 & 12.7 & 11. & & 26 & 24 & 1.16 & 1.19 & .28 & 1.14 \\
\hline 41 & agg2 & 642 & 72 & 72 & 34 & 769 & 215 & 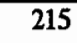 & 125 & 4. & 1. & 1. & 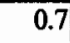 & 15 & 72 & 1.72 & 6.72 & 1.53 & 1.53 \\
\hline 42 & agg? & 614 & 71 & 71 & 36 & 73 & 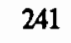 & & 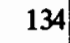 & 4. & 1. & 1. & 0.7 & .46 & 1.80 & 1.80 & 6.65 & 1.77 & 1.77 \\
\hline 43 & $\operatorname{ses} d 6$ & 22 & 22 & 22 & 11 & 58 & 58 & $\pi$ & 42 & 1. & 1. & 1. & 1.4 & 38 & 1.38 & 1.38 & 1.23 & 1.23 & 1.23 \\
\hline 44 & ship04 & 413 & 34 & 34 & 18 & 5 & 48 & & 2 & 2.0 & 1 & 1 & 1.2 & .95 & 1.75 & 1.75 & 1.68 & 1.45 & 1.45 \\
\hline 45 & seba & 659 & 659 & 659 & 138 & 853 & 853 & 3 & 234 & 5 & 5. & 5.0 & 1.4 & 65 & 3.65 & 3.65 & 3.56 & 3.56 & 3.56 \\
\hline 46 & tuff & 377 & 384 & 384 & 366 & 474 & 428 & 428 & 427 & 1.9 & 2.0 & 2.0 & 2.4 & 11 & 1.00 & 1.00 & 0.83 & 0.83 & 0.83 \\
\hline 47 & forpl & 32 & ? & $3 ?$ & 166 & 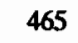 & 472 & . & 336 & 1. & 1. & 1.6 & 1.3 & 38 & 1.40 & 1.40 & .14 & .21 & 1.21 \\
\hline 48 & bn & 22 & 2147 & 2147 & 24 & & & & & & & 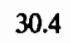 & 366 & 15 & 0.90 & 0.90 & 0.90 & 0.83 & 0.83 \\
\hline 49 & pilot4 & 1063 & 851 & 729 & 322 & 1744 & 1549 & 1334 & 1015 & 26.5 & 22.4 & 18.8 & 14.6 & 1.72 & 1.53 & 1.31 & 1.82 & 1.54 & 1.29 \\
\hline 50 & scfxm2 & 858 & 602 & 602 & 400 & 1116 & 884 & 884 & 669 & 9.3 & 6.3 & 6.3 & 5.3 & 1.67 & 1.32 & 1.32 & 1.74 & 1.17 & 1.17 \\
\hline
\end{tabular}




\section{TABLE V}

Comparison of Initial Bases:

Artificial (A), Feasible Slack (FS), Slack (S), CPLEX (C)

\begin{tabular}{|c|c|c|c|c|c|c|c|c|c|c|c|c|c|c|c|c|c|c|c|}
\hline & \multicolumn{4}{|c|}{ Phase I Iterations } & \multicolumn{4}{|c|}{ Total Iterations } & \multicolumn{4}{|c|}{$\begin{array}{c}\text { Run Times } \\
\text { (seconds) }\end{array}$} & \multicolumn{3}{|c|}{\begin{tabular}{|c|}
$\begin{array}{c}\text { Total Iterations } \\
\text { Ratios }\end{array}$ \\
\end{tabular}} & \multicolumn{3}{|c|}{$\begin{array}{c}\text { Run Time } \\
\text { Ratios } \\
\end{array}$} \\
\hline & & $\mathbf{A}$ & FS & $\mathbf{S}$ & C & $\mathbf{A}$ & FS & $\mathbf{S}$ & $\mathbf{C}$ & $\mathbf{A}$ & FS & $\mathbf{s}$ & $\mathbf{C}$ & $\mathrm{A} / \mathrm{C}$ & FS/C & $\mathbf{S} / \mathbf{C}$ & $\mathrm{A} / \mathrm{C}$ & $\mathrm{FS} / \mathrm{C}$ & $\mathrm{S} / \mathrm{C}$ \\
\hline 51 & row15 & 0 & 0 & 0 & 0 & 849 & 849 & 849 & 621 & 11.8 & 11.8 & 11.8 & $\overline{11.3}$ & 1.37 & 1.37 & 1.37 & 1.04 & 1.04 & 1.04 \\
\hline 52 & perold & 3614 & 3298 & 3428 & 1370 & 4629 & 4416 & 4679 & 2457 & 100.1 & 94.3 & 102.9 & 52.3 & 1.88 & 1.80 & 1.90 & 1.91 & 1.80 & 1.97 \\
\hline 53 & ffffrso0 & 1106 & 1058 & 917 & 541 & 1290 & 1220 & 1064 & 683 & 7.4 & 7.1 & 5.9 & 4.5 & 1.89 & 1.79 & 1.56 & 1.63 & 1.58 & 1.30 \\
\hline 54 & ship041 & 383 & 360 & 360 & 223 & 565 & 525 & 525 & 353 & 2.2 & 2.1 & 2.1 & 1.6 & 1.60 & 1.49 & 1.49 & 1.32 & 1.30 & 1.30 \\
\hline 5 & sctap2 & 1356 & 679 & 611 & 252 & 2328 & 1655 & 1505 & 548 & 29.0 & 20.1 & 18.2 & 4.7 & 4.25 & 3.02 & 2.75 & 6.24 & 4.31 & 3.92 \\
\hline 56 & ganges & 1591 & 1579 & 1579 & 358 & 1714 & 1727 & 1727 & 605 & 21.1 & 21.7 & 21.7 & 9.6 & 2.83 & 2.85 & 2.85 & 2.21 & 2.27 & 2.27 \\
\hline 5 & ship08s & 772 & 697 & 697 & 202 & 1073 & 955 & 955 & 429 & 7.5 & 6.7 & 6.7 & 3.6 & 2.50 & 2.23 & 2.23 & 2.07 & 1.85 & 1.85 \\
\hline 58 & sierra & 2175 & 577 & 577 & 206 & 3068 & 841 & 841 & 455 & 36.3 & 7.2 & 7.2 & 4.4 & 6.74 & 1.85 & 1.85 & 8.20 & 1.62 & 1.62 \\
\hline 59 & scfxm3 & 1240 & 906 & 906 & 596 & 1681 & 1280 & 1280 & 1050 & 18.8 & 12.3 & 12.3 & 11.6 & 1.60 & 1.22 & 1.22 & 1.62 & 1.06 & 1.06 \\
\hline 60 & ship12s & 1108 & 1102 & 1102 & 316 & 1346 & 1287 & 1287 & 528 & 12.6 & 11.5 & 11.5 & 6.3 & 2.55 & 2.44 & 2.44 & 1.99 & 1.82 & 1.82 \\
\hline 61 & grow 22 & 0 & 0 & 0 & 0 & 1224 & 1224 & 1224 & 892 & 23.5 & 23.5 & 23.5 & 21.8 & 1.37 & 1.37 & 1.37 & 1.08 & 1.08 & 1.08 \\
\hline 62 & stocfor 2 & 2657 & 1503 & 1503 & 411 & 3508 & 2182 & 2182 & 1061 & 78.6 & 46.0 & 40.0 & 26.5 & 3.31 & 2.06 & 2.06 & 2.97 & 1.74 & 1.74 \\
\hline 63 & $\operatorname{scsd} 8$ & 1039 & 1039 & 1039 & 468 & 2023 & 2023 & 2023 & 1320 & 12.0 & 12.0 & 12.0 & 8.2 & 1.53 & 1.53 & 1.53 & 1.47 & 1.47 & 1.47 \\
\hline 64 & sctap3 & 1703 & 951 & 869 & 337 & 2896 & 2273 & 2221 & 786 & 46.5 & 40.0 & 39.1 & 9.2 & 3.68 & 2.89 & 2.83 & 5.04 & 4.33 & 4.24 \\
\hline 65 & pilotwe & 1618 & 1626 & 1626 & 675 & 3533 & 3737 & 3737 & 2652 & 75.5 & 83.4 & 83.4 & 76.2 & 1.33 & 1.41 & 1.41 & 0.99 & 1.09 & 1.09 \\
\hline 66 & maros & 1215 & 742 & 742 & 760 & 1912 & 1367 & 1367 & 1476 & 22 & 15.7 & 7 & 18.8 & 1.30 & 0.93 & 0.93 & 1.19 & 0.84 & 0.84 \\
\hline 6 & fitlp & 837 & 837 & 837 & 450 & 1249 & 1249 & 1249 & 809 & 16.8 & 16.8 & 16.8 & 11.2 & 1.54 & 1.54 & 1.54 & 1.49 & 1.49 & 1.49 \\
\hline 68 & $25 \mathrm{fv} 47$ & 1760 & 1414 & 1377 & 979 & 3734 & 3438 & 3439 & 2679 & 94 & 76.3 & 77.6 & 65.1 & 1.39 & 1.28 & 1.28 & 1.44 & 1.17 & 1.19 \\
\hline 65 & czprob & 1421 & 1245 & 1245 & 547 & 2321 & 2083 & 2083 & 1178 & 17.8 & 16.6 & 16.6 & 10.8 & 1.97 & 1.77 & 1.77 & 1.65 & 1.53 & 1.53 \\
\hline 70 & ship081 & 771 & 732 & 732 & 326 & 1387 & 1283 & 1283 & 804 & 10.3 & 9.6 & 9.6 & 6.6 & 1.73 & 1.60 & 1.60 & 1.56 & 1.46 & 1.46 \\
\hline 71 & pilotnov & 2864 & 2701 & 2614 & 2262 & 3161 & 3228 & 3237 & 2767 & 69.5 & 74.8 & 75.8 & 71.2 & 1.14 & 1.17 & 1.17 & 0.98 & 1.05 & 1.06 \\
\hline 72 & nesm & 1777 & 1951 & 1951 & 961 & 5029 & 5307 & 5307 & 4094 & 36.6 & 38.2 & 38.2 & 31.0 & 1.23 & 1.30 & 1.30 & 1.18 & 1.23 & 1.23 \\
\hline 73 & fitld & 0 & 0 & 0 & 0 & 1161 & 989 & 989 & 947 & 4.7 & 4.0 & 4.0 & 3.9 & 1.23 & 1.04 & 1.04 & 1.21 & 1.05 & 1.05 \\
\hline 74 & bnl2 & 4640 & 4051 & 4051 & 2858 & 6819 & 5564 & 5564 & 4422 & 250.5 & 186.0 & 186.0 & 148.2 & 1.54 & 1.26 & 1.26 & 1.69 & 1.25 & 1.25 \\
\hline 75 & pilotja & 4583 & 3259 & 3543 & 2011 & 7065 & 5459 & 6004 & 4365 & 207.1 & 157.7 & 182.0 & 139.0 & 1.62 & 1.25 & 1.38 & 1.49 & 1.13 & 1.31 \\
\hline 76 & ship121 & 1220 & 1147 & 1147 & 579 & 1648 & 1502 & 1502 & 969 & 18.3 & 15.8 & 15.8 & 12.5 & 1.70 & 1.55 & 1.55 & 1.46 & 1.26 & 1.26 \\
\hline 77 & cycle & 0 & 0 & 0 & 0 & 2416 & 2034 & 2034 & 1207 & 38.6 & 32.2 & 32.2 & 27.1 & 2.00 & 1.69 & 1.69 & 1.42 & 1.19 & 1.19 \\
\hline 78 & 80 bau3b & 4056 & 2195 & 2227 & 2227 & 11107 & 10173 & 10635 & 10635 & 173.8 & 163.0 & 166.2 & 166.2 & 1.04 & 0.96 & 1.00 & 1.05 & 0.98 & 1.00 \\
\hline 79 & degen 3 & 3188 & 2964 & 3107 & 2873 & 5471 & 4860 & 5043 & 4633 & 254.7 & 202.0 & 204.5 & 201.4 & 1.18 & 1.05 & 1.09 & 1.26 & 1.00 & 1.02 \\
\hline 80 & truss & 3482 & 3482 & 3482 & 2205 & 11511 & 11511 & 11511 & 10622 & 206.8 & 206.8 & 206.8 & 192.4 & 1.08 & 1.08 & 1.08 & 1.07 & 1.07 & 1.07 \\
\hline 81 & greenbea & 3688 & 3473 & 3473 & 2966 & 7582 & 8115 & 8115 & 7973 & 282.8 & 320.3 & 320.3 & 341.5 & 0.95 & 1.02 & 1.02 & 0.83 & 0.94 & 0.94 \\
\hline 82 & greenbeb & 3695 & 3661 & 3661 & 3171 & 6717 & 7037 & 7037 & 6121 & 250.5 & 268.5 & 268.5 & 241.8 & 1.10 & 1.15 & 1.15 & 1.04 & 1.11 & 1.11 \\
\hline 83 & d2q06c & 4778 & 3770 & 2944 & 1427 & 10922 & 10258 & 9832 & 9649 & 684.4 & 668.6 & 655.8 & 684.6 & 1.13 & 1.06 & 1.02 & 1.00 & 0.98 & 0.96 \\
\hline 84 & woodw & 1099 & 1026 & 1113 & 369 & 2791 & 2160 & 2440 & 1701 & 38.1 & 28.6 & 31.3 & 25.5 & 1.64 & 1.27 & 1.43 & 1.50 & 1.12 & 1.23 \\
\hline 85 & pilots & 5495 & 4552 & 4530 & 4034 & 8599 & 8039 & 7698 & 7402 & 822.7 & 833.1 & 757.8 & 768.3 & 1.16 & 1.09 & 1.04 & 1.07 & 1.08 & 0.99 \\
\hline 86 & fit $2 p$ & 5271 & 5271 & 5271 & 3076 & 12585 & 12585 & 12585 & 10030 & 796.0 & 796.0 & 796.0 & 690.5 & 1.25 & 1.25 & 1.25 & 1.15 & 1.15 & 1.15 \\
\hline 87 & stocfor 3 & 21140 & 12220 & 12220 & 5015 & 28698 & 18443 & 18443 & 10813 & 4288.5 & 2762.6 & 2762.6 & 1817.5 & 2.65 & 1.71 & 1.71 & 2.36 & 1.52 & 1.52 \\
\hline 88 & wood1p & 211 & 211 & 211 & 222 & 459 & 459 & 459 & 749 & 8.7 & 8.7 & 8.7 & 14.0 & 0.61 & 0.61 & 0.61 & 0.62 & 0.62 & 0.62 \\
\hline 89 & pilot87 & 5213 & 4211 & 4633 & 4053 & 10835 & 10296 & 9995 & 9696 & 2179.0 & 2176.9 & 2105.1 & 2087.2 & 1.12 & 1.06 & 1.03 & 1.04 & 1.04 & 1.01 \\
\hline 90 & fit2d & 2 & 0 & 0 & 0 & 12593 & 12041 & 12041 & 11726 & 131.1 & 125.0 & 125.1 & 120.0 & 1.07 & 1.03 & 1.03 & 1.09 & 1.04 & 1.04 \\
\hline & $\begin{array}{l}\text { TALS } \\
\text { ERAGES }\end{array}$ & 122926 & 94758 & 4384 & 8249 & 226982 & 197485 & 97434 & 157966 & 11572 & 9752 & 9627 & 8276 & .09 & 1.55 & 1.5 & 1.90 & 1.36 & 1.35 \\
\hline
\end{tabular}




\section{Acknowledgement}

The author would like to thank Bob Fourer for teaching him how to think about the simplex method and Tom Baker for motivating the development of the CPLEX code. Thanks are also due to John Gregory for a number of helpful comments on the original version of this manuscript.

\section{References}

[1] Adler, I., N. Karmarkar, M. G. C. Resende, and G. Veiga (1989), An implementation of Karmarkar's algorithm for linear programming, Mathematical Programming 44 No. 3, 297-336.

[2] Chvátal, Vašek (1983), Linear Programming, W. H. Freeman, New York.

[3] Dantzig, G. B. (1951), Maximization of a linear function of variables subject to linear inequalities, in : Activity Analysis of Production and Allocation, R.C. Koopmans (ed.), Wiley, New York, 1951, 339-347.

[4] Golub, G. H. and C. F. Van Loan (1983), Matrix Computations, The Johns Hopkins University Press, Baltimore, Maryland.

[5] Optimization Subroutine Library: Guide and Reference (1990), IBM Corporation, April 1990.

[6] Karmarkar, N. (1984), A new polynomial time algorithm for linear programming, Combinatorica 4, 375-395.

[7] Lustig, I. (1987), An analysis of an available set of linear programming test problems, Technical Report SOL 87-11, Systems Optimization Lab, Stanford University, Stanford, California.

[8] Lustig, I. (1987), An analysis of an available set of linear programming test problems, Comput. Opns Res. 16 173-184.

[9] Lustig, I. J., R. E. Marsten, and D. F. Shanno (1990), On implementing Mehrotra's predictor-corrector interior-point method for linear programming, Technical Report SOR 90-03, Department of Civil Engineering and Operations Research, Princeton University, Princeton, NJ.

[10] Markowitz, H. M. (1957), The elimination form of the inverse and its application to linear programming, Management Science 3 255-269.

[11] Murtagh, Bruce A. and Michael Saunders (1987), Minos 5.1 User's Guide, Technical Report SOL 83-20R, Systems Optimization Lab, Stanford University, Stanford, California. 
[12] Suhl, Uwe H. and Leena M. Suhl (1990), Computing sparse LU factorizations for large-scale linear programming bases, ORSA Journal on Computing 2 325-335. 